\title{
CONSERVATION OF ENDANGERED ENDEMIC SEABIRDS WITHIN A MULTI-PREDATOR CONTEXT: THE BARAU'S PETREL IN RÉUNION ISLAND
}

\author{
Y. DUMONT* \\ CIRAD, Umr AMAP, Montpellier, France, \\ E-mail: yves.dumont@cirad.fr
}

J. C. RUSSELL

Department of Environmental Science, Policy and Management, University of California, Berkeley, CA

\section{LECOMTE}

CNRS, Centre d'études biologique de Chizé, Beauvoir-sur-Niort, France

\author{
M. LE CORRE
}

Ecomar, Université de la Réunion, St Denis, France

\begin{abstract}
Seabirds breeding on islands are vulnerable to introduced predators, such as rats and cats, and the removal of such predators is generally viewed as a priority for seabird conservation and restoration. However, multiple invasive mammal species interacting may generate unexpected outcomes following the removal (eradication) of one species. Generally these indirect interactions are not well understood or demonstrated. We propose and study a prey (seabird)mesopredator (rat)-superpredator (cat) model, taking into account the juvenile stages in the prey population, in order to direct conservation management for seabird conservation. We give a more biologically realistic differential system than those studied before (Courchamp et al. [1999]; Fan et al. [2005]), in particular for long-lived seabird species. We present a theoretical study and show existence and uniqueness of a positive solution as well as a qualitative study of the equilibria that may appear. Because standard numerical methods, usually implemented in scientific softwares, can fail to give the right biological approximations (Anguelov et al. [2009]), we propose a reliable algorithm that preserves most of the qualitative properties of the continuous system, using the theory of nonstandard finite difference methods. Finally, we use biologically realistic parameters available for the representative Barau's petrel (Pinet et al. [2008]), an endemic species from
\end{abstract}

\footnotetext{
*Corresponding author: Y. Dumont; CIRAD, Umr AMAP, Montpellier, France, e-mail: yves.dumont@cirad.fr.

Received by the editors on $31^{\text {st }}$ October 2009. Accepted $13^{\text {th }}$ May 2010. 


\begin{abstract}
Réunion island, to present numerical simulations that support the theoretical study. Cats play the major role in seabird prey population dynamics. Seasonality in seabird breeding delays but does not prevent extinction. In all scenarios, cat control (or preferably eradication) is imperative to prevent extinction of vulnerable long-lived seabirds, like the Barau's petrel.
\end{abstract}

KEY WorDs: Population dynamics, intraguild predation, mesopredator, superpredator, equilibrium, stability/instability, nonstandard finite difference method, dynamic consistency, numerical simulation.

1. Introduction. Globally biodiversity is undergoing a dramatic decline, attributable entirely to the impact of humans (Vitousek et al. [1997]). Conservation and management of natural ecosystems has become an important and necessary task in order to preserve as much as possible of the biological diversity in many places around the world Le Corre [2008]. This is particularly so on oceanic islands, which house a disproportionate amount of the world's biodiversity relative to their area. Isolated islands have also been disproportionately impacted by the introduction of non-native species. Indeed, many studies have now shown that introductions of predators, like rats and cats, have driven to extinction many endemic insular species. For example, the introduction of alien species is now recognized as directly or indirectly responsible for $42 \%$ of bird extinctions on islands (King [1985]). In response it is necessary and urgent to develop control or eradication programs to restore island ecosystems (e.g., Howald et al. [2007]). In that way, mathematics has become a powerful tool giving prospective insight into the dynamics of interacting populations, helping conservation managers make informed and appropriate choices about the outcomes of control programmes.

Réunion Island is a small French overseas department, located in the South-West of Indian Ocean, and is known to be a hot spot of biological diversity in the world. Réunion Island has been inhabited for three and a half centuries, and with a growing human population, the challenge is to preserve its endemic heritage. Some endemic, and endangered, seabirds and land birds are still living on Réunion Island. Unfortunately, we know that many endemic birds have become extinct since the arrival of humans on Réunion Island. Thus, the aim is now to preserve as much as possible of the remaining endemic bird populations.

Introduced cats (Felis catus) (Robertson [2008]) and rats (Rattus $s p p$ ) are two of the most widespread invasive mammal taxa on oceanic 
islands, directly impacting bird populations through opportunistic and omnivorous predation. The isolation of insular bird populations has often caused a lack of evolutionary response to mammalian predation, meaning they are usually preferentially preyed upon (Pearre and Maass [1998]). Seabirds are particularly vulnerable, requiring a terrestrial phase for breeding where most species are ground nesting and poorly adapted to locomotion. Cats and rats have made easy prey of such seabirds, and for declining populations pest control or eradication is often imperative.

We are mainly concerned with intraguild predation: when cats (a superpredator) prey upon rats (a mesopredator) and they both share a prey species, a specific case of "mesopredator release effect" has been hypothesized. In this scenario the eradication of one predator may alter the dynamics of the system depending on the strength of trophic relationships. For example the eradication of cats may cause a population increase in rats, which will change the impact rats have at the population level on birds. Although intraguild predation (Müller and Brodeur [2002]) has been observed often, mesopredator release effect (of cats and rats) has never actually been demonstrated. It has been mathematically described previously using ordinary logistic differential equations for a simple cat-rat-bird system (Courchamp et al. [1999]), cat-rat-bird system with functional response (Fan et al. [2005]), and cat-rat-bird system with spatial heterogeneity (Gambino et al. [2007]). In all cases the authors have reached identical conclusions that the removal of cats will cause an increase in rats that will negatively affect the bird species of conservation concern; a counter intuitive conclusion that cat eradication does not benefit birds. However, in all cases the authors simplistically assumed that all bird species on an island could be treated as an "average" and that the impacts of cats and rats were identical in form except in the numerical (population size) level of impact. These studies also used rapid growth rates typical only of short-lived high-fecundity ( $r$-selected) bird species such as forest passerines. We believe that this modeling is not adapted to long-lived seabirds, like the Barau's petrel or other long-lived species.

Because oceanic island bird conservation usually focuses on seabird species (e.g., Jones et al. [2008]), and complete eradication of introduced cats and rats is now possible (Nogales et al. [2004]; Howald et al. [2007]), we focus on the case of cats-rats-seabirds. 
Functionally seabirds differ substantially from land birds. They are much larger and longer-lived ( $K$-selected), including a subadult atsea phase where they are immune to terrestrial predation. Seabirds also differ in their breeding biology, in the most extreme case laying only a single egg clutch with high parental investment. They also lack behavioral plasticity such as to change breeding location when predation risks increase (Igual et al. [2007]). Because of these differences the impact of cats and rats on seabirds is not identical, violating the implicit assumption of previous studies (Courchamp et al. [1999]; Fan et al. [2005]; Gambino et al. [2007]). Cats are large predators $(<2.5$ $\mathrm{kg}$ ), which can impact both the chicks and breeding adults of small to medium-sized seabirds (Pearre and Maass [1998]); whereas invasive rats $(<0.5 \mathrm{~kg})$ can generally only impact the eggs and chicks due to their smaller size (Jones et al. [2008]). These size-mediated predation impacts of cats and rats on an age-structured seabird population can be graphically conceptualized (see Figure 1).

A critical assumption of the mesopredator release model dynamic is the presence of alternative prey for the predators at an arbitrary fixed abundance. We include alternative prey for both rats and cats, allowing realistic ecosystem dynamics even in the absence (e.g., extinction) of seabirds, as considered by Fan et al. [2005] but treated differently. Introduced cats and rats are omnivorous and highly plastic in their feeding behavior, enabling persistence on many islands despite poor resources. This assumption is likely to be met on most islands where vegetation, insects, or small reptiles can provide an alternative and abundant prey item but would have important consequences, which we do not explore if this alternative prey item was also a species of conservation concern (e.g., Caut et al. [2008]).

The rest of the paper is organized as follows. In Section 2, we present the general cat-rat-seabird model. Then, in Section 3, we give some theoretical results. In particular, we derive some results about the local (global) stability/instability of the equilibria. In Section 4, we use the nonstandard finite difference approach to construct a numerical scheme that preserves as much as possible the properties of the continuous system. Using data obtained for the Barau's petrel (Pinet et al. [2008]), an endangered seabird endemic to Réunion Island, we present some numerical results that illustrate the theoretical part. Finally, in Section 5 we discuss the 


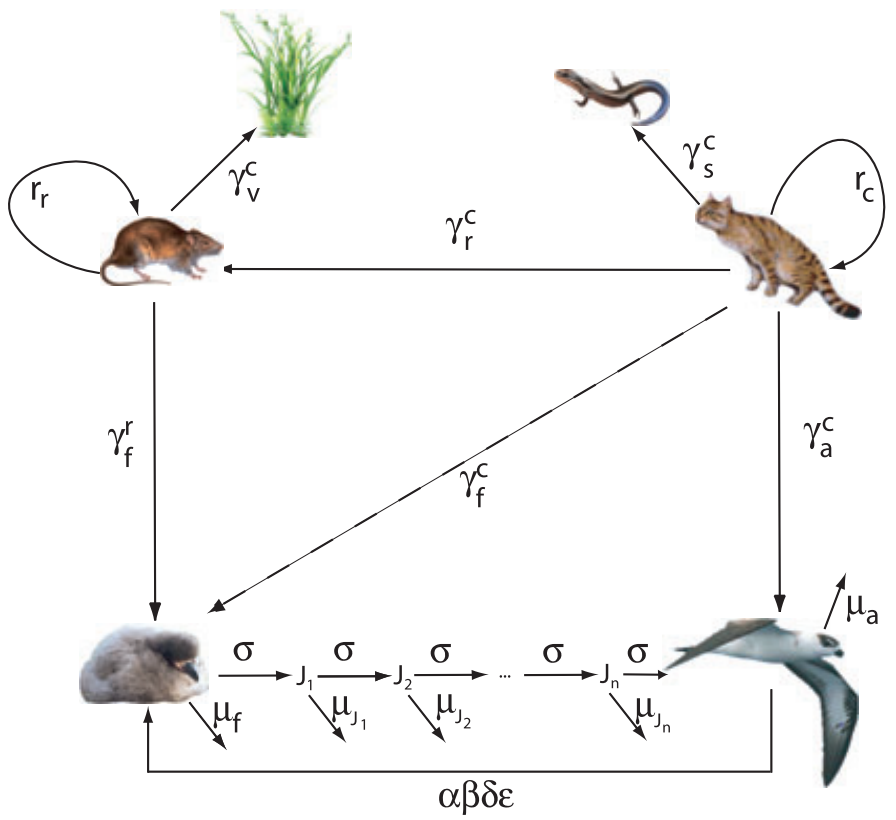

FIGURE 1. Conceptual representation of the age-structured differential predation model. $\gamma_{j}^{i}$ is the predation rate of population $i$ on population $j$ where $c=$ cats, $r=$ rats, $f=$ fledglings, $a=$ adult birds, and $v=$ vegetation. $r_{i}$ is the annual intrinsic growth rate of population $i$. For birds, $\alpha, \beta, \delta$, and $\epsilon$ are the sex-ratio, adult breeding proportion, adult pair fecundity, and number of clutches, respectively, $\mu_{f}$ is fledgling mortality, $\mu_{j_{k}}$ is juvenile mortality for age class $k$, for $n$ sub-adult age classes, and $\mu_{a}$ is adult mortality. In our parameterisation sub-adults (age classes $J_{1} \cdots J_{n}$ ) are seabirds remaining at sea and are only subjected to "natural" mortality.

broader conservation implications of our results, and, in particular, we show that without control on cat populations, seabirds can disappear in a very short time. In a companion piece of work (Russell et al. [2009]), we consider through simulation the nonequilibrium dynamics of a comparable model with respect to community regulation on predator population dynamics. Here, we focus on the impact of control strategies for seabird conservation and provide full mathematical proofs. 
2. The model. The model is illustrated in Figure 1.

For the bird population we describe three distinct life stages; fledgling, juveniles (nonbreeding), and adult (breeding). Juveniles progress through $n$ age classes equivalent to annual transitions. The number of individuals in each age class is given by $F, J_{k}$, and $A$, respectively. No senescence is assumed, and time is measured in annual increments, given the annual nature of breeding in many seabird species.

The fledgling age class is modeled by the density-dependent reproduction of adults, minus those birds leaving the current age class, either through mortality or aging, minus those preyed upon by rats and cats (both relative to the amount of alternative prey available).

The juvenile age-classes 1 through $n$ are simply those surviving from the previous age class minus those leaving the current age class. We assume that these birds are immune to predation by either cats or rats, given that these individuals have progressed beyond the predationprone fledgling size class and do not engage in predation-prone nesting. This is especially true for juvenile seabirds, which spend the majority of their time at sea except for occasional prenesting prospecting. The only source of mortality for these birds is at sea (e.g., natural or fisheries-related mortality).

The adult age class is similarly modeled by those surviving from the previous juvenile age class, minus those adults who die from "natural" (nonpredation) causes minus those that are preyed upon by cats (relative to the amount of alternative prey available).

Hence, for the entire bird population:

$$
\left\{\begin{aligned}
\frac{d F}{d t}= & \alpha \beta \delta \epsilon A\left(1-\frac{F}{K_{f}}\right)-\mu_{f} F-\sigma_{f} F-\frac{F}{F+V} \gamma_{f}^{r} R \\
& -\frac{F}{F+A+R+S} \gamma_{f}^{c} C, \\
\frac{d J_{k}}{d t}= & \sigma_{j_{k-1}} J_{k-1}-\mu_{j_{k}} J_{k}-\sigma_{j_{k}} J_{k}, \quad \text { for } k=1, \ldots, n \\
\frac{d A}{d t}= & \sigma_{j_{n}} J_{n}-\mu_{a} A-\frac{A}{F+A+R+S} \gamma_{a}^{c} C,
\end{aligned}\right.
$$

where $K_{f}$ is fixed at the total number of available nest sites; $F, J_{k}, A, R, C, V$, and $S$ are the population size of fledglings, juvenile 
age-class $k$ (with $J_{0}=F$ ), adult birds, rats, cats, and alternative rat and cat food, respectively; $\alpha, \beta, \delta$, and $\epsilon$ are the sex-ratio, adult breeding proportion, adult pair fecundity, and number of clutches, respectively. $\mu_{i}$ and $\sigma_{i}$ are mortality and survival of age-class $i\left(F, J_{k}, A\right)$, respectively. $\gamma_{j}^{i}$ is the predation rate (number of prey per predator) of $i$ (cats and rats) on $j$ (rats, adults, fledglings, and vegetation). For simplification, we consider from now on that $\sigma_{0}=\sigma_{F}, \sigma_{j_{k}}=\sigma$, and $\mu_{j_{k}}=\mu$, for all $k=1, \ldots, n$, i.e., survival and mortality rates are constant across all juvenile age classes.

For the predators we use typical logistic population growth models for the entire population, following Courchamp et al. [1999]. Both species can breed in their first year, and so there is no need to distinguish age classes. We assume that size (growth-related) variation within predator species does not alter their predation rates. Rats are modeled by the density-dependent growth rate minus those that die naturally and that are controlled and minus their predation by cats (relative to the number of cats and amount of alternative prey available). Cats are modeled by the density-dependent growth rate minus those that die naturally minus those that are controlled.

Hence for the predators, we have the following system

$$
\left\{\begin{array}{l}
\frac{d R}{d t}=r_{r} R\left(1-\frac{R}{K_{r}}\right)-\tau_{r} R-\frac{R}{F+A+R+S} \gamma_{r}^{c} C, \\
\frac{d C}{d t}=r_{c} C\left(1-\frac{C}{K_{c}}\right)-\tau_{c} C,
\end{array}\right.
$$

where $r_{i}$ and $K_{i}$ are the annual intrinsic growth-rate and carrying capacity (see later) of species $i, \tau_{i}$ is the removal rate (simulated control effort) of species $i$, and $\gamma_{j}^{i}$ are as stated earlier. $\tau$ is interpreted as the proportional decay in the growth rate, $r$, of each predator population as control is undertaken.

Carrying capacities for all three species are:

$$
\begin{aligned}
& K_{f}=\text { fixed } \\
& K_{r}=\frac{F}{\gamma_{f}^{r}}+\frac{V}{\gamma_{v}^{r}} \text { for } \gamma_{j}^{i}>0 \\
& K_{c}=\frac{A}{\gamma_{a}^{c}}+\frac{F}{\gamma_{f}^{c}}+\frac{R}{\gamma_{r}^{c}}+\frac{S}{\gamma_{s}^{c}} \text { for } \gamma_{j}^{i}>0
\end{aligned}
$$


where $K_{r}$ and $K_{c}$ are functions of the available prey and their rates of consumption.

We use the ODE's approach like Courchamp et al. [1999] and Fan et al. [2005] in order to be able to compare our results with those obtained in the aforementioned papers. But, of course, several other approaches can be used to model the system cat-ratseabird. For instance, it could be possible to consider a discrete system or a first-order finite difference system, like $X_{n+1}=F\left(X_{n}\right)$. Another approach would be to consider delay differential equations. In that case the subsystem (1) could be reduced, for instance, to

$$
\begin{aligned}
\frac{d F(t)}{d t}= & \alpha \beta \delta \epsilon A(t)\left(1-\frac{F(t)}{K_{f}}\right)-F(t)-\frac{F(t)}{F(t)+V} \gamma_{f}^{r} R(t) \\
& -\frac{F(t)}{F(t)+A(t)+R(t)+S} \gamma_{f}^{c} C(t), \\
\frac{d A(t)}{d t}= & F(t-\tau) e^{-\sigma \tau}-\mu_{a} A(t)-\frac{A(t)}{F(t)+A(t)+R(t)+S} \gamma_{a}^{c} C(t),
\end{aligned}
$$

where $F(t-\tau) e^{-\sigma \tau}$ represents the rate of maturation of surviving fledgings to adulthood; $\sigma$ is the constant mortality rate of the juvenile, $\tau$ represents the length of the delay, in years, for a fledging to become an adult. Age-structured matrix population models also provide an alternative approach, which we do not consider (e.g., Igual et al. [2009]). Unfortunately, from the mathematical point of view, the previous approaches are more difficult to handle than the standard ODE's approach, in particular for large coupled nonlinear systems.

3. The system seabird-rat-cat. We now consider system (1)-(2) with appropriate initial conditions. The right-hand side of the system is Lipschitz continuous, and thus from classical analysis we deduce that there exists a unique maximal solution. Note also that system (1)-(2) 
can be summarized as follows

$$
\left\{\begin{array}{l}
\frac{d x}{d t}=\mathcal{M}(x) x ; \\
x(0)=x_{0},
\end{array}\right.
$$

with $\quad x=\left(F, J_{1}, \ldots, J_{n}, A, R, C\right)^{T} \in \mathbb{R}_{+}^{n+4} \quad$ and $\quad \mathcal{M}(x) \quad$ a square matrix.

If $\tau_{c}<r_{c}$ and $\tau_{r}<r_{r}$, we can define the following compact subset $\quad$ of $\quad \mathbb{R}_{+}^{n+4}: \quad \mathcal{G}=\left\{\left(F, J_{1}, \ldots, J_{n}, A, R, C\right) \in \mathbb{R}_{+}^{n+4} / F \leq K_{f}, J_{k} \leq\right.$ $\left.\frac{\sigma_{f} \sigma^{k-1}}{\mu_{a}} K_{f}, A \leq \sigma_{f} \sigma^{n} K_{f}, R \leq\left(1-\frac{\tau_{r}}{r_{r}}\right) K_{r, \text { max }}, C \leq\left(1-\frac{\tau_{c}}{r_{c}}\right) K_{c, \text { max }}\right\}$, where the upper bound $K_{r, \max }$ and $K_{c, \max }$ are defined as follows

$$
\begin{aligned}
& K_{r, \text { max }}=\frac{K_{f}}{\gamma_{f}^{r}}+\frac{V}{\gamma_{v}^{r}}, \\
& K_{c, \max }=\left(\frac{\sigma_{f} \sigma^{n}}{\mu_{a} \gamma_{a}^{c}}+\frac{1}{\gamma_{f}^{c}}\right) K_{f}+\frac{K_{r, \max }}{\gamma_{r}^{c}}+\frac{S}{\gamma_{s}^{c}} .
\end{aligned}
$$

It can be verified that $\mathcal{G}$ is positively invariant with respect to the system (1)-(2). Altogether, we deduce

Theorem 1. Let $\left(F(0), J_{1}(0), \ldots, J_{n}(0), A(0), R(0), C(0)\right) \in \mathcal{G}$, then there exists a unique positive solution in $\mathcal{G}$.

We will now provide some results about the existence of equilibria and their qualitative properties. We consider the following three cases:

(i) the seabird only, without predators;

(ii) the seabird with only one predator, the rat;

(iii) the seabird with two predators, the rat and the cat.

Some general definitions and useful results are recalled in Appendix A, and proofs are given in full detail in the next appendices 
3.1. The system without predators. First, we consider the previous systems without rats and cats. Thus we consider (4), with $R=C=0$ and we obtain the following (sub)system

$$
\left\{\begin{array}{c}
\frac{d X}{d t}=\mathcal{A}(X) X ; \\
X(0)=X_{0},
\end{array}\right.
$$

with $X=\left(F, J_{1}, \ldots, J_{n}, A\right)^{T} \in \mathcal{G}^{-}$, and $\mathcal{G}^{-}$a compact subset of $\mathbb{R}_{+}^{n+3}$ defined by

$$
\begin{aligned}
\mathcal{G}^{-} & =\left\{\left(F, J_{1}, \ldots, J_{n}, A,\right)\right. \\
& \left.\in \mathbb{R}_{+}^{n+2} / 0 \leq F \leq K_{f}, 0 \leq J_{k} \leq \sigma_{f} \sigma^{k-1} K_{f}, 0 \leq A \leq \sigma_{f} \sigma^{n} K_{f}\right\}
\end{aligned}
$$

and

$(6) \mathcal{A}(X)=\left(\begin{array}{cccccc}-\left(1+\alpha \beta \delta \varepsilon \frac{A}{K_{f}}\right) & 0 & \cdots & 0 & 0 & \alpha \beta \delta \varepsilon \\ \sigma_{f} & -1 & 0 & \ddots & 0 & 0 \\ 0 & \sigma & -1 & 0 & \ddots & 0 \\ \vdots & \ddots & \ddots & \ddots & \ddots & 0 \\ \vdots & \ddots & \ddots & \sigma & -1 & 0 \\ 0 & \cdots & \cdots & 0 & \sigma & -\mu_{a}\end{array}\right)$.

Remark 1. For all $X \in \mathbb{R}_{+}^{n+3}, \mathcal{A}(X)$ is a Metzler matrix, i.e. a matrix such that off diagonal terms are nonnegative Thus the positive orthant $\mathbb{R}_{+}^{n+2}$ is positively invariant, which means that if $X(0) \geq 0$, then $X(t) \geq 0$ for all time $t>0$.

Let us define

$$
\mathcal{R}=\frac{\alpha \beta \delta \epsilon \sigma^{n} \sigma_{f}}{\mu_{a}}
$$


In demographic terms, $\mathcal{R}$ is the basic offspring number of the bird population.

\section{Proposition 1. System (5) has}

(i) a trivial equilibrium $E_{0}=(0, \ldots, 0)$.

(ii) a feasible biological equilibrium $E_{*}=\left(F^{*}, J_{1}^{*}, \ldots, J_{n}^{*}, A^{*}\right)$ if and only if $\mathcal{R}>1$.

Proof. Taking $\frac{d X}{d t}=0$ in (5) leads to

$$
\left\{\begin{array}{l}
A^{*}=\frac{\sigma}{\mu_{a}} J_{n}^{*}, \\
J_{n}^{*}=\sigma J_{n-1}^{*}, \\
\vdots \\
J_{1}^{*}=\sigma_{f} F^{*}, \\
F^{*}=\alpha \beta \delta \varepsilon A^{*}\left(1-\frac{F^{*}}{K_{f}}\right)
\end{array}\right.
$$

We deduce that $A^{*}=\frac{\sigma_{f}}{\mu_{a}} \sigma^{n} F^{*}$ and replacing in (8) leads to

$$
F^{*}=\alpha \beta \delta \varepsilon \frac{\sigma_{f}}{\mu_{a}} \sigma^{n} F^{*}\left(1-\frac{F^{*}}{K_{f}}\right) .
$$

We deduce either $F^{*}=0$ and we get the trivial equilibrium or $F^{*}>0$, with

$$
F^{*}=\left(1-\frac{\mu_{a}}{\alpha \beta \delta \varepsilon \sigma^{n} \sigma_{f}}\right) K_{f}=\left(1-\frac{1}{\mathcal{R}}\right) K_{f},
$$

if and only if $\mathcal{R}>1$. Then, using (7) and (8), $A^{*}, J_{1}^{*}, \ldots$, and $J_{n}^{*}$ follow.

Then we prove the following

\section{Proposition 2.}

- If $\mathcal{R} \leq 1$, the seabird population will go extinct, i.e., $E_{0}$ is globally asymptotically stable. 
- If $\mathcal{R}>1$, the seabird population can reach the nonzero equilibrium $E_{*}$, i.e., $E_{*}$ is locally asymptotically stable.

Proof. See Appendix B.

3.2. The system seabird-rat. Like in the previous section, it is possible to rewrite (4), with $C=0$, in the following manner

$$
\left\{\begin{array}{c}
\frac{d X}{d t}=\mathcal{B}(X) X ; \\
X(0)=X_{0},
\end{array}\right.
$$

where $X=\left(F, J_{1}, \ldots, J_{n}, A, R\right)^{T} \in \mathbb{R}^{n+3}$ and

$$
\begin{aligned}
& \mathcal{B}(X)
\end{aligned}
$$

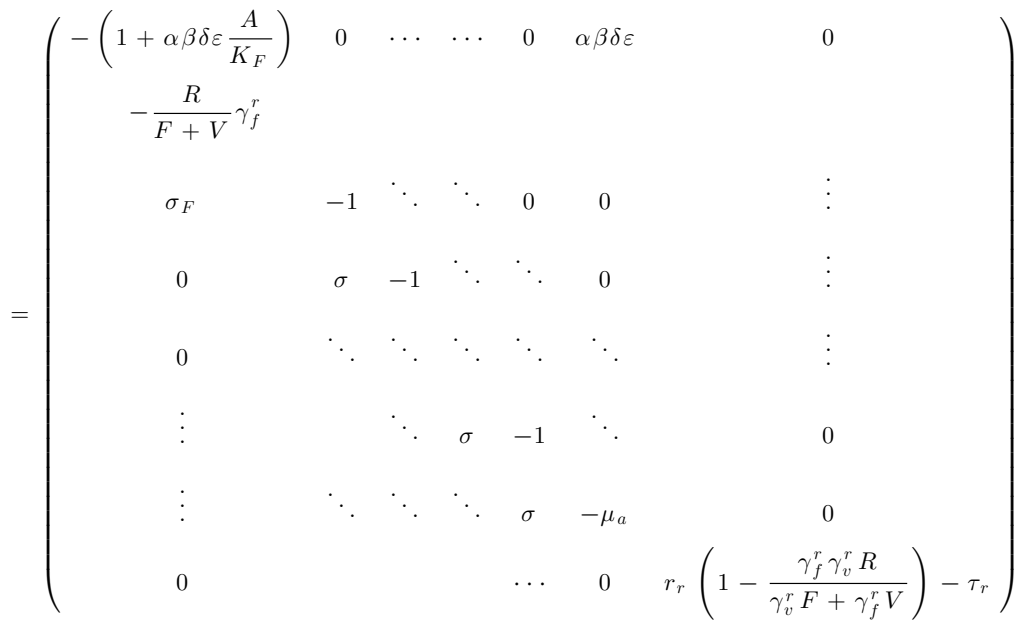

We set

$$
\mathcal{R}_{\tau_{r}}=1+\frac{\gamma_{f}^{r}}{\gamma_{v}^{r}}\left(1-\frac{\tau_{r}}{r_{r}}\right)
$$

Then we prove 
Proposition 3. For the system seabirds-rats there are four possible theoretical equilibria:

(i) the trivial equilibrium, $E_{0}=(0, \ldots, 0)$.

(ii) If $r_{r}>\tau_{r}$ and $\mathcal{R}<1$, then $E_{1}=\left(0, \ldots, 0, R^{*}\right)$ with $R^{*}=(1-$ $\left.\frac{\tau_{r}}{r_{r}}\right) \frac{V}{\gamma_{v}^{r}}$.

(iii) If $r_{r}<\tau_{r}$ and $\mathcal{R}>1$, then $E_{*}=\left(F^{*}, J_{1}^{*}, \ldots, J_{n}^{*}, A^{*}, 0\right)$, where $F^{*}, \ldots, A^{*}$ are given in (8) and (7),

(iv) If $r_{r}>\tau_{r}$ and $\mathcal{R}>\mathcal{R}_{\tau_{r}}$, then $E_{*}^{r}=\left(F^{r}, J_{1}^{r}, \ldots, J_{n}^{r}, A^{r}, R^{r}\right)$, where $F^{r}$ is given in (A3), and $J_{1}^{r}, \ldots, A^{r}$ and $R^{r}$ are given in (A2).

Proof. See Appendix C.

Then we show the following results about the long-term dynamic of the system seabird-rat.

\section{Proposition 4 (see Table 1).}

(i) If $r_{r}<\tau_{r}$ and $\mathcal{R}<1$, then the rat and the seabird populations can go extinct, i.e., the equilibrium $E_{0}$ is locally asymptotically stable.

(ii) If $r_{r}>\tau_{r}$ and $0<\mathcal{R}<\mathcal{R}_{\tau_{r}}$, then the seabird population can go extinct and the rat population can reach a positive equilibrium, i.e., $E_{1}$ is locally asymptotically stable.

(iii) If $r_{r}<\tau_{r}$ and $1<\mathcal{R}$, then the rat population can go extinct and the seabird population can reach a positive equilibrium, i.e., $E_{*}$ is locally asymptotically stable

(iv) If $r_{r}>\tau_{r}$ and $\mathcal{R}>\mathcal{R}_{\tau_{r}}$, then the rat and seabird populations can reach the positive equilibrium $E_{*}^{r}$, i.e., $E_{*}^{r}$ is locally asymptotically stable.

Proof. See Appendix D.

Remark 2. From item 2 in Proposition 4, it is clear, that if the rat predation on fledgings is important, i.e., $\gamma_{f}^{r} \gg \gamma_{r}^{v}$, with $1<\mathcal{R}$, then the seabird population can go extinct. In that case, it would be necessary to have a strong rat control to prevent the extinction of the seabird. 
TABLE 1. Existence, stability/instability of the equilibrium points for the sub system seabird-rat.

\begin{tabular}{lllcc}
$\mathcal{R}$ & $\tau_{r}$ & Existence & Stable & Instable \\
\hline$<1$ & $>r_{r}$ & $E_{0}$ & $E_{0}$ & - \\
$<\mathcal{R}_{\tau_{r}}$ & $<r_{r}$ & $E_{0}, E_{1}$ & $E_{1}$ & $E_{0}$ \\
$>1$ & $>r_{r}$ & $E_{0}, E_{*}$ & $E_{*}$ & $E_{0}$ \\
$>\mathcal{R}_{\tau_{r}}$ & $<r_{r}$ & $E_{0}, E_{*}, E_{*}^{r}$ & $E_{*}^{r}$ & $E_{0}, E_{*}$ \\
\hline
\end{tabular}

In the Table 1, we summarize some of the previous results.

3.3. The full system: seabird-rat-cat. We now consider system (4), and we show the following:

Proposition 5. System (4) admits the following equilibria:

(i) the trivial equilibrium $E_{0}=(0, \ldots, 0,0)$,

(ii) $E^{*}=\left(F^{*}, J_{1}^{*}, \ldots, J_{n}^{*}, A^{*}, 0,0\right)$, where $F^{*}, J_{1}^{*}, \ldots$, and $A^{*}$ are given in Proposition 3 , if $\mathcal{R}>1$.

(iii) $E_{1}^{*}=\left(0, \ldots, 0, R^{*}, 0\right)$, where $R^{*}$ is given in Proposition 3, if $r_{r}>$ $\tau_{r}, r_{c}<\tau_{c}$ and $\mathcal{R}<\mathcal{R}_{\tau_{r}}$.

(iv) $E^{* *}=\left(F^{r}, J_{1}^{r}, \ldots, J_{n}^{r}, A^{r}, R^{r}, 0\right)$, where $F^{r}, J_{1}^{r}, \ldots, A^{r}$ and $R^{r}$ are defined in Proposition $3_{4}$, if $r_{r}>\tau_{r}$ and $\mathcal{R}>\mathcal{R}_{\tau_{r}}$.

(v) $E^{* * *}=\left(0, \ldots, 0, R^{*}, C^{*}\right)$, if $r_{c}>\tau_{c}$ and $r_{r}>\tau_{r}$.

Proof. See appendix E.

The previous results give various indications on the long-term behavior of the system.

Remark 3. Equilibrium $E_{1}^{*}$ corresponds to the well known mesopredator release effect: If cat control is large enough, then the cat population declines and thus an increase of the rat population occurs such that if $\mathcal{R}$ is not sufficiently large, the seabird can go extinct. The mesopredator release effect is not automatic when the cat disappears: 
it should depend on the basic offspring number too. In particular if $\mathcal{R}>\mathcal{R}_{\tau_{r}}$ there will be no mesopredator release effect because the intrinsic growth of the seabird population will compensate the impact of rat predation.

Remark 4. Note also that if $\frac{\tau_{r}}{r_{r}}<1$, the size of interval $\left[1, \mathcal{R}_{\tau_{r}}\right]$ depends on rat predation on fledgings, i.e., if $\gamma_{f}^{r} \gg \gamma_{v}^{r}$ (see (10)). Thus the estimates of predation rates are particularly important.

Remark 5. Through long but straighforward computations, it is possible to show that a positive equilibrium cat-seabird $\left(F^{*}, J_{1}^{*}, \ldots, A^{*}, 0, C^{*}\right)$ can exist "mathematically" (we are able to compute it) but does not belong to the compact subset $\mathcal{G}$ and thus is not biologically realistic (see Appendix F).

Finally, it is possible to show that a "full" cat-rat-seabird equilibrium can exist. Unfortunately, it is not possible to solve analytically the system (A5), but, numerically, it is possible to give the following formal proposition.

Proposition 6. Suppose that $\tau_{r}=0$. There exists a positive real, $\tau_{*}$ (the level of cat control), such that if $0<\tau_{*} \leq \tau_{c}<r_{c}$, an equilibrium cat-rat-seabird, $E_{f u l l}=\left(F^{*}, J_{1}^{*}, \ldots, A^{*}, R^{*}, C^{*}\right)$ exists, i.e., biologically realistic.

When rat control is considered, the previous proposition can be extended (numerically) in the following manner

Proposition 7. Let $\tau_{r, *}$ be a positive real such that $\tau_{r, *} \geq r_{r}$ and suppose $\tau_{r} \in\left[0, \tau_{r, *}\right]$. Then, there exists a positive real, $\tau_{*}$, that may depend on $\tau_{r}$ such that if $0<\tau_{*} \leq \tau_{c}<r_{c}$, an equilibrium cat-rat-seabird, $E_{f u l l}=\left(F^{*}, J_{1}^{*}, \ldots, A^{*}, R^{*}, C^{*}\right)$, exists, i.e., biologically realistic and locally stable.

The previous results indicate clearly that there is no viable equilibrium between seabirds, rats, and cats without appropriate controls on the predators.

In Figures 8 and 9, we show the evolution of the equilibrium with respect to the control parameters, $\tau_{c}$ and $\tau_{r}$.

Finally, it is possible to show the following result about the long-term dynamic of the full system 


\section{Proposition 8 (see Table 2).}

(i) If $r_{r}>\tau_{r}$ or/and $r_{c}>\tau_{c}$, the full system will never reach the equilibrium $E^{*}=\left(F^{*}, J_{1}^{*}, \ldots, J_{n}^{*}, A^{*}, 0,0\right)$ or the equilibrium $E^{* *}=$ $\left(F^{*}, J_{1}^{*}, \ldots, J_{n}^{*}, A^{*}, R^{*}, 0\right)$.

(ii) If $r_{r}<\tau_{r}, r_{c}<\tau_{c}$ and $\mathcal{R}>1$, then the full system will reach, at least locally, the equilibrium $E^{*}=\left(F^{*}, J_{1}^{*}, \ldots, J_{n}^{*}, A^{*}, 0,0\right)$.

(iii) if $r_{r}>\tau_{r}, r_{c}<\tau_{c}$ and $\mathcal{R}>\mathcal{R}_{\tau_{r}}$, then the full system will reach, at least locally, the equilibrium $E^{* *}=\left(F^{r}, J_{1}^{r}, \ldots, J_{n}^{r}, A^{r}, R^{r}, 0\right)$.

(iv) If $r_{r}>\tau_{r}$ and $r_{c}>\tau_{c}$, then the full system will reach, at least locally, the equilibrium $E^{* * *}=\left(0,0, \ldots, 0, R^{*}, C^{*}\right)$.

Proof. See Appendix G.

The previous proposition shows that without controls and with $\mathcal{R}>1$ the whole system can only reach the equilibrium $E^{* * *}$; in that case the seabird population disappears while the rat and the cat populations reach a nonzero equilibrium. When cat and rat controls are introduced, the long-term behavior of the system becomes more complicated: If the level of control is high enough, i.e., such that cats and rats are eradicated, then the seabird population will reach its original equilibrium. If the controls are not strong enough, then we can reach different equilibrium depending on the values taken by the control parameters $\tau_{c}$ and $\tau_{r}$.

In Table 2, we summarize some of the previous results

Altogether, the system can develop a complex dynamic, and this dynamic can change according to the parameters. Thus depending on the parameters, the long-term behavior indicates that the seabird population can go extinct or reach an equilibrium with the rat population. A full equilibrium cat-rat-seabird seems possible but only for a narrow range of parameters, and this make this equilibrium difficult to reach in reality. The theory show us that without a sufficient control on cats preferably, seabird populations have very little chance of survival.

In the next section we explain the numerical scheme we use in our computations. Then, using the parameters available for the Barau's petrel, we will present some numerical simulations to illustrate our theoretical results. 


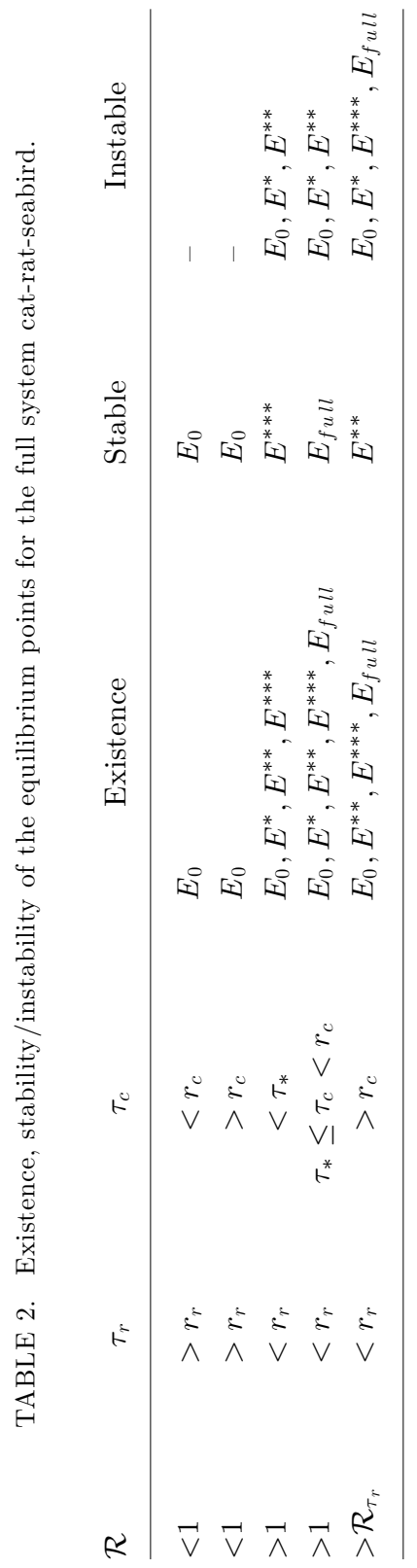


Computations with the routine ODE implemented in Scilab

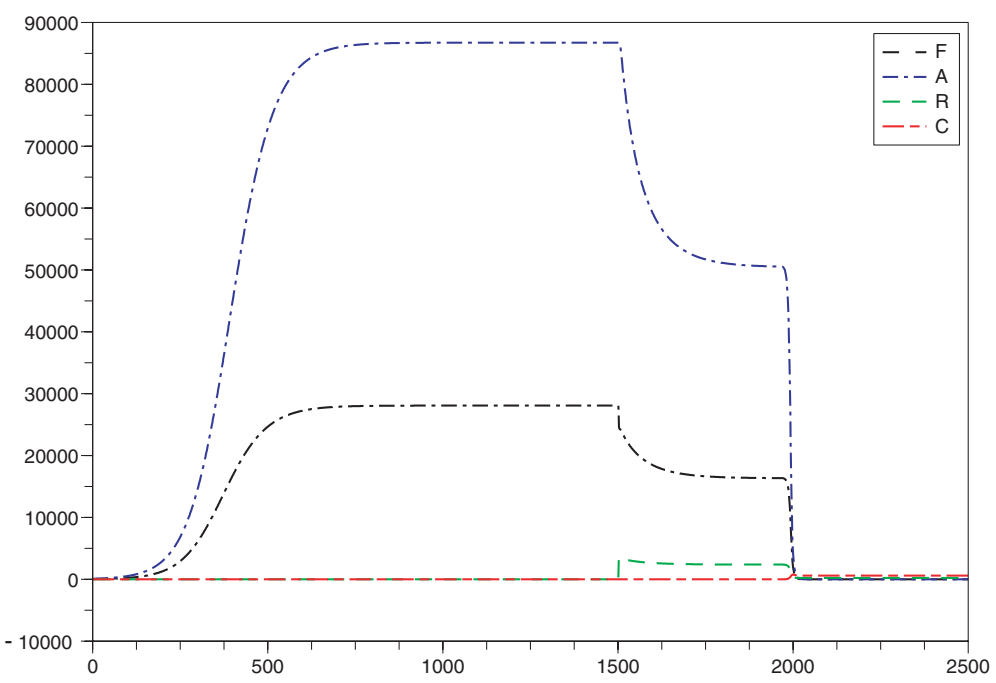

FIGURE 2. Example of spurious negative solutions obtained with the ODE's routine implemented in Scilab.

\section{Construction of a suitable algorithm. Numerical simulations.}

4.1. A dynamic consistent algorithm. Let $X_{n}$ be an approximation of $X\left(t_{n}\right)$ where $t_{n}=n \Delta t$, with $n \in \mathbb{N}$.

In the previous section, we have presented different properties of the theoretical solution and the problems that we may take into account in our numerical scheme. Compartmental models are usually solved using standard numerical methods, for example, Euler or Runge Kutta methods. Unfortunately, these methods can lead to negative solutions, exhibit numerical instabilities, or even converge to the wrong equilibrium for certain values of the time discretization or the model parameters (see Anguelov et al. [2009] for further investigations). For instance, in Figure 2, using the parameters, we show that a "naive use" of the 
ODE's routine implemented in Scilab can exhibit negative solutions (compare also with Figure 5a). Thus, the use or the construction of a dynamical consistent scheme is a crucial step, and it seems that this step is often of minor concern in ecology. Here, we aim to present some simple rules to construct a scheme that preserves most of the properties of the continuous system.

Nonstandard methods (Mickens [1994], [2002]) have shown great potential in many areas, like ecology, epidemiology, and other disciplines (see for instance Anguelov et al. [2009]; Dimitrov and Kojouharov [2006]; Gumel et al. [2005]; Mickens [2000, 2005]; Moghadas et al. [2003]). Thus, we aim to construct a robust scheme that preserves most of the properties of the solution of the continuous problem. We require our scheme to be dynamically consistent, in the sense that the numerical solution should reproduce any property of the exact solution and/or of the differential system. To do that, we follow Mickens' rules to obtain a nonstandard numerical scheme (see Mickens [1994, 2000, 2002, 2005] and references therein).

We also use Mickens' rules: the standard denominator in the discrete denominator is replaced by a time-step function $0<\Phi(\Delta t)<1$, such that $\Phi(\Delta t)=O(\Delta t)$; then, we approximate the nonlinear terms in a nonlocal way and consider the linear terms in an implicit way. Thus a nonstandard approximation scheme for the system (1)-(2) is given by

$$
\left\{\begin{array}{l}
\frac{F^{n+1}-F^{n}}{\Phi(\Delta t)}=\alpha \beta \varepsilon \delta A^{n+1} \\
\quad-\left(1+\alpha \beta \varepsilon \delta \frac{A^{n}}{K_{F}}+\frac{1}{F^{n}+V} \gamma_{f}^{r} R^{n}+\frac{1}{F^{n}+A^{n}+R^{n}+S} \gamma_{f}^{c} C^{n}\right) F^{n+1}, \\
\frac{J_{k}^{n+1}-J_{k}^{n}}{\Phi(\Delta t)}=-J_{k}^{n+1}+\sigma J_{k-1}^{n+1}, \quad \text { for } k=1, \ldots, n \text { and } J_{0}^{n}=F^{n}, \\
\frac{A^{n+1}-A^{n}}{\Phi(\Delta t)}=\sigma J_{N}^{n+1}-\left(\mu_{a}+\frac{1}{F^{n}+A^{n}+R^{n}+S} \gamma_{a}^{c} C^{n}\right) A^{n+1},
\end{array}\right.
$$


and

$$
\left\{\begin{array}{l}
\frac{R^{n+1}-R^{n}}{\Phi(\Delta t)}=r_{r} R^{n+1} \\
-\left(\tau_{r}+r_{r} \frac{R^{n}}{K_{r}}+\frac{1}{F^{n}+A^{n}+R^{n}+S} \gamma_{r}^{c} C^{n}\right) R^{n+1} \\
\text { with } K_{r}=\frac{F^{n}}{\gamma_{f}^{r}}+\frac{V}{\gamma_{v}^{r}} \\
\quad \text { with } K_{c}=\frac{A^{n}}{\gamma_{a}^{c}}+\frac{F^{n}}{\gamma_{f}^{c}}+\frac{R^{n}}{\gamma_{r}^{c}}+\frac{S}{\gamma_{v}^{c}}
\end{array}\right.
$$

Altogether, we can rewrite the previous systems in the following matrix formulation, using (4)

$$
\left(I d-\Phi(\Delta t) \mathcal{M}\left(X^{n}\right)\right) X^{n+1}=X^{n} .
$$

Thus, it suffices to choose $\Phi(\Delta t)$ such that $I d-\Phi(\Delta t) \mathcal{M}\left(X^{n}\right)$ is an $M$-matrix, for all $\Delta t>0$, which implies that $\left(I d-\Phi(\Delta t) \mathcal{M}\left(X^{n}\right)\right)^{-1} \geq 0$, for all $\Delta t>0$. In fact, we have $I d-\Phi(\Delta t) \mathcal{M}\left(X^{n}\right)=$

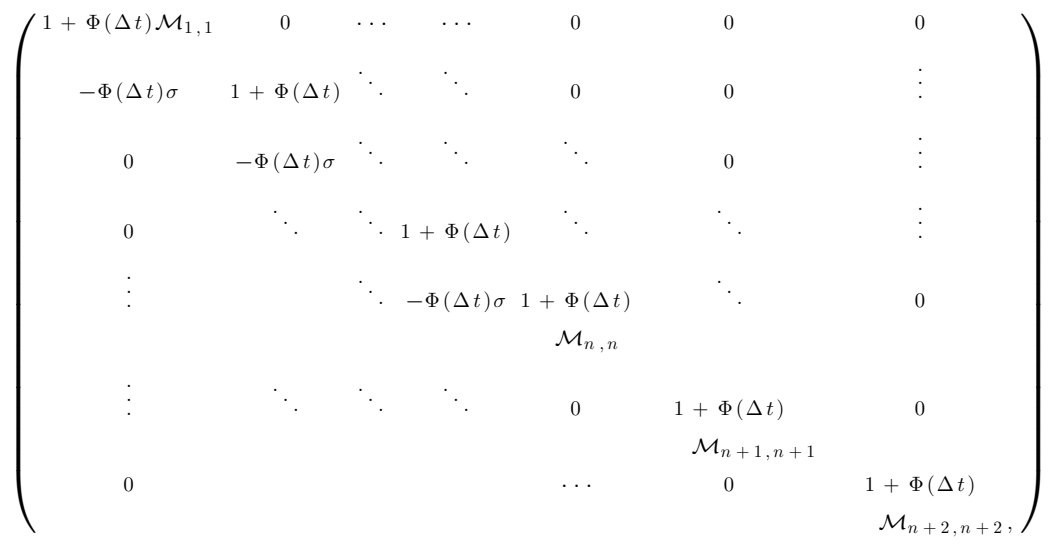


with

$$
\begin{aligned}
\mathcal{M}_{1,1}(X)= & (1-\alpha \beta \delta \varepsilon)+\alpha \beta \delta \varepsilon \frac{A}{K_{F}}+\frac{R}{F+V} \gamma_{f}^{r} \\
& +\frac{1}{F+A+R+S} \gamma_{f}^{c} C, \\
\mathcal{M}_{n, n}(X)= & \mu_{a}+\frac{1}{F+A+R+S} \gamma_{a}^{c} C, \\
\mathcal{M}_{n+1, n+1}(X)= & \tau_{r}+r_{r}\left(\frac{\gamma_{f}^{r} \gamma_{v}^{r} R}{\gamma_{v}^{r} F+\gamma_{f}^{r} V}-1\right)+\frac{1}{F+A+R+S} \gamma_{r}^{c} C, \\
\mathcal{M}_{n+2, n+2}(X)= & \tau_{c}+r_{c}\left(\frac{C}{K_{c}}-1\right) .
\end{aligned}
$$

Thus, following Theorem 5 (in Appendix A), it suffices to choose $\Phi(\Delta t)$ such that $1+\Phi(\Delta t) M_{11}>0$ and $1+\Phi(\Delta t) M_{n+1, n+1} \geq 0$, which implies that $\mathcal{M}^{T}\left(X^{n}\right)$ is diagonaly dominant, and thus $\mathcal{M}\left(X^{n}\right)$ is an $M$-matrix. In particular, the following time-step function is convenient

$$
\Phi(\Delta t)=\frac{1-e^{-Q \Delta t}}{Q},
$$

with

$$
Q \geq \max \left(r_{r}-\tau_{r}, r_{c}-\tau_{c}\right) .
$$

It is also clear that, for any positive initial data, we obtain positive approximations that are located in the feasible region. Our scheme is positively stable for all $\Delta t>0$. Critical points or equilibrium of the continuous problem are solutions of $\mathcal{M}\left(X_{e}\right) X_{e}=0$. Thus multiplying by $\Phi(\Delta t)$ and summing $X_{e}$ in both sides, leads to

$$
\left(I d-\Phi(\Delta t) \mathcal{M}\left(X_{e}\right)\right) X_{e}=X_{e},
$$

which clearly shows that $X_{e}$ is a fixed point of (13) also. Thus the numerical scheme and the continuous problem have the same equilibria, which are assumed to be hyperbolic. We now intend to show that our scheme is elementary stable, i.e., the scheme (13) preserves the local stability/instability property of any equilibrium. 
The dynamics of the model (1)-(2) can be captured by any number $Q$ that satisfies

$$
Q \geq \max \left\{|\lambda|^{2} / 2|\operatorname{Re} \lambda|\right\},
$$

where $\lambda$ traces the eigenvalues of the Jacobian matrices of the righthand side of model (1)-(2) at any equilibrium. Thus we have the following result.

Theorem 2. The NSFD scheme (13) is elementary stable whenever $\Phi(\Delta t)$ is chosen according to (14), (15), and (16).

Proof. We have shown that the NSFD scheme (13) has no extra fixed points than the equilibrium points of (1)-(2). Let $X^{*}$ denote the equilibrium of the system (1)-(2). Let $J \equiv J_{\mathcal{M}}\left(X^{*}\right)$ be the Jacobian matrix of the right-hand side of system (1)-(2) at $X^{*}$, i.e., $J_{i j}=\frac{\partial M_{i}}{\partial X_{j}}$. $J$ is in practice diagonalizable, using the factorization

$$
\Lambda^{-1} J \Lambda=\operatorname{diag}\left(\lambda_{1}, \lambda_{2}, \ldots \lambda_{n}\right),
$$

where $\Lambda$ is a transition matrix. Thus, setting $\varepsilon=X-X^{*}$, the linearization of the system (1)-(2) at $X^{*}$ reads as

$$
\frac{d \varepsilon}{d t}=J \varepsilon,
$$

which is equivalent to the uncoupled system

$$
\frac{d \eta}{d t}=\operatorname{diag}\left(\lambda_{1}, \lambda_{2}, \ldots \lambda_{n}\right) \eta .
$$

Thus, applying the NSFD scheme (13) to the system (17) or (18), we obtain the linearized schemes

$$
\varepsilon^{k+1}=(I-\Phi(\Delta t) J)^{-1} \varepsilon^{k}
$$

or

$$
\eta^{k+1}=\operatorname{diag}\left(\frac{1}{1-\Phi(\Delta t) \lambda_{1}}, \ldots, \frac{1}{1-\Phi(\Delta t) \lambda_{n}}\right) \eta^{k}
$$


Now if $X^{*}$ is asymptotically stable for (1)-(2), then the real parts of all the eigenvalues $\lambda_{i}$ are negative, and it follows from (19) that

$$
\begin{aligned}
\rho & \left((I-\Phi(\Delta t) J)^{-1}\right) \\
& =\max \left\{\frac{1}{\left|1-\Phi(\Delta t) \lambda_{i}\right|} ; 1 \leq i \leq n\right\} \\
& =\max \left\{\frac{1}{\sqrt{1+2 \Phi(\Delta t)\left|\operatorname{Re} \lambda_{i}\right|+\Phi^{2}(\Delta t)\left|\lambda_{i}\right|^{2}}} ; 1 \leq i \leq n\right\} \\
& <1,
\end{aligned}
$$

which shows that $X^{*}$ is asymptotically stable for the scheme (13).

Suppose now that $X^{*}$ is unstable for (1)-(2). Then there exists at least one eigenvalue of $J$, say $\lambda_{1}$, with a positive real part. We then have

$$
\begin{aligned}
\frac{1}{\left|1-\Phi(\Delta t) \lambda_{1}\right|^{2}} & =\frac{1}{1-2 \Phi(\Delta t) \operatorname{Re} \lambda_{1}+\Phi^{2}(\Delta t)\left|\lambda_{1}\right|^{2}} \\
& >1
\end{aligned}
$$

whenever the requirement (16) is met. Therefore, $X^{*}$ is unstable for the scheme (13). Thus, at least locally, we are sure that the scheme (13) has the same dynamic as the system (1)-(2).

Q.E.D.

Altogether the numerical algorithm or the finite difference system (13) is said to be dynamically consistent with the continuous system. This is of utmost importance in particular because we are interested in the long-term behavior of the system.

4.2. Numerical simulations. In order to test the applicability of any model, it is imperative to calibrate it with biologically appropriate and empirical data. Appropriate demographic data can often be difficult to collect however, and many models often fit "inappropriate" data that may not reflect biological realities. We test our model with recent data collected on the breeding biology and differential predation upon an endemic seabird species, the Barau's petrel Pterodroma baraui of Réunion Island (western Indian Ocean). The Barau's petrel is a member of the family Procellariidae and of the gadfly petrel group (Pterodroma spp.). This genus is characterized by a global 
distribution, with high rates of endemism on oceanic islands. The genus is highly phylogenetically constrained with very little variation in breeding biology and growth rates. Adult females lay only one egg each year, with high natal site fidelity and prolonged incubation and chick rearing ( 5 months). Adults are long lived (decades) and do not recruit immediately as breeding adults ( $5+$ years), instead spending their juvenile phase at-sea occasionally returning to prospect around their birth site. The Barau's petrel population is estimated between 4,000 to 6,500 pairs, which annually nest above $2,400 \mathrm{~m}$ (Le Corre et al. [2002]). In this respect it is similar to the Hawaiian dark-rumped petrel $(P$. sandwichensis; Simons [1984]). Barau's petrel are in decline (Probst et al. [2000]) and urgent conservation action is required. In particular, recent diet analysis of cats and rats has been used to parameterize predation rates of cats and rats on Barau's petrels (Faulquier et al. [2008]). The data available in Table 3 provide a rich source in conjunction with our model to test different cat and rat control options (eradication is not feasible) in order to save the Barau's petrel.

In particular with the data given in Table 3 , we always verify $\mathcal{R}>1$. Thus, from the theoretical result, we know that the system will never reach some of the mathematical equilibria studied in the theoretical section. In the following simulations, we always consider $\Delta t=1$ (year).

We simulate a scenario similar to Réunion Island considering the dynamics of petrels without predation, i.e., without rats and cats: in Figure 3 , we show that petrel dynamics tend slowly to the nontrivial equilibrium, $E_{*}$. Then, in Figure 4a, we suppose that rats were introduced in the year 1500, when Arabian navigators first knew about the Mascarene islands (Réunion Island, Mauritius, and Rodrigues). We show that the whole system reaches a new equilibrium, $E_{* *}$, as expected from theory (see Propositions 3 and 4 ). This shows that predation by rats is not sufficient to cause extinction of the bird population. Similarly, in Figure 4b, we suppose that cats have only recently expanded into high-altitude breeding colonies of Barau's petrel at the same time as human development around $t=1970$. As expected from theory, there is no viable positive equilibrium and the petrel population rapidly disappears.

Finally, we simulate a scenario similar to the colonization of Reunion Island by introducing first rats and then cats (see Figure 5a). In this 
TABLE 3. Model parameterization for mesopredator release in an age-structured bird population for Barau's petrel.

Parameter

Symbol Long-lived large (petrel)

Adult sex-ratio

$\begin{array}{cc}\alpha & 0.5 \\ \beta & 0.9 \\ \delta & 1 \\ \epsilon & 1 \\ n & 5 \\ \mu_{F} & 0.34 \\ \mu_{J k} & 0.2 \\ \mu_{a} & 0.07 \\ E(\omega) & 18 \\ m a x(\omega) & 48 \\ r_{B} & 0.03 \\ \lambda_{B}\left(e^{r_{B}}\right) & 1.04 \\ K_{F} & 100,000 \\ r_{r} & 4 \\ r_{c} & 0.25 \\ \tau_{r} & 0 \\ \tau_{c} & 0(0.24,0.25,0.26,0.30)\end{array}$

Proportion of adults breeding

Adult pair fecundity

Number of clutches

Sub-adult classes

Fledgling mortality

Sub-adult annual mortality

Adult mortality

Expected adult life time ${ }^{\mathrm{a}}$

Maximum adult life time ${ }^{\mathrm{a}}$

Annual bird growth rate ${ }^{\mathrm{a}}$

Bird annual reproduction

Adult bird carrying capacity

Annual rat growth rate ${ }^{\mathrm{a}}$

Annual cat growth rate ${ }^{a}$

Rats control rate

Cats on rats

$\gamma_{r}^{c}$

Cats on adult birds

$\gamma_{a}^{c}$

22

Cats on fledglings

$\gamma_{f}^{c}$

22

Rats on fledglings

$\gamma_{f}^{r}$

8

Rats on alternative (vegetation)

$\gamma_{v}^{r}$

300

Cats on alternative (skinks)

$\gamma_{s}^{c}$

168

Alternative food available for rats

V

100,000

Alternative food available for cats

$S$

Source

Faulquier et al. [2008];

Simons [1984]; de la

Brooke [1995]

${ }^{a}$ Derived. 
Petrels: time evolution of the population

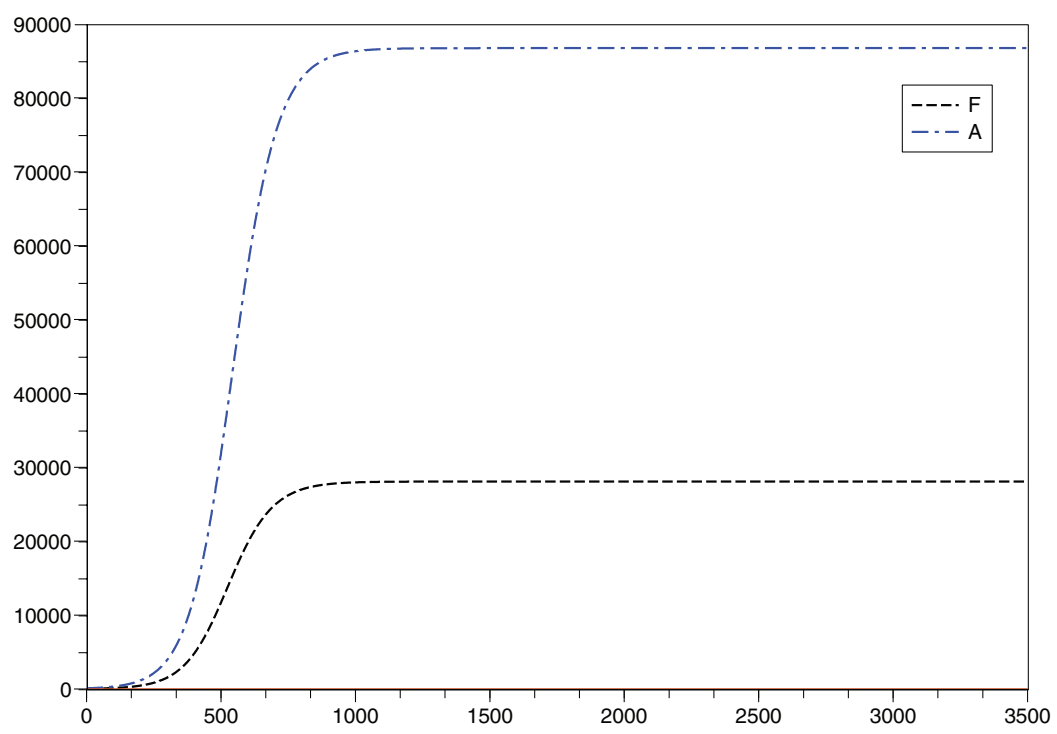

FIGURE 3. Dynamics of the petrel population (F for fledglings, A for adults).

scenario, with $\tau_{r}=\tau_{c}=0$, we show that the population of Barau's petrel will rapidly decline to extinction in less than one century (Figure 5b) while, at the same time, rats and cats will reach an equilibrium, i.e., the system reaches equilibrium $E^{* * *}$, as expected from the theory. Our previous theoretical results and the numerical experiments showed that medium-sized seabirds could coexist with rats but not with cats. We now investigate cat control measures but within a multipredator context, which specifically considers potential mesopredator release of rats, in order to estimate the optimal levels of control and recovery time for the seabird population.

In Figure 6, we show the population dynamics for different values of $\tau_{c}$, around its equilibrium level $(0.23,0.24,0.25$, and 0.30$)$. As long as $\tau_{c}<\tau_{*}$, the system will never reach the equilibrium $E^{* * *}$. We show numerically that $\tau_{*} \approx 0.235576$, such that $E_{\text {full }}$ appears when $\tau_{c}$ belongs in a very small interval $[0.235576,0.25]$; see also Figure $6 \mathrm{a}$. The time to 


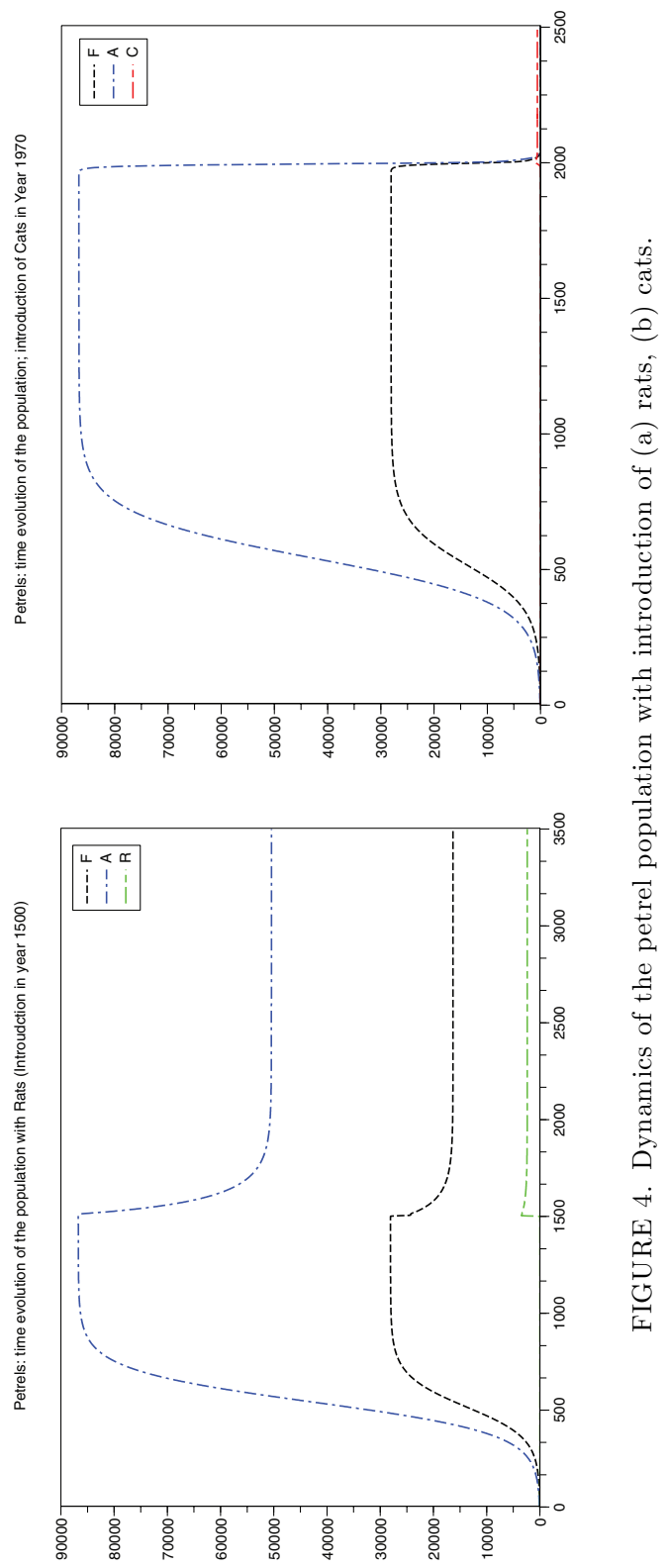



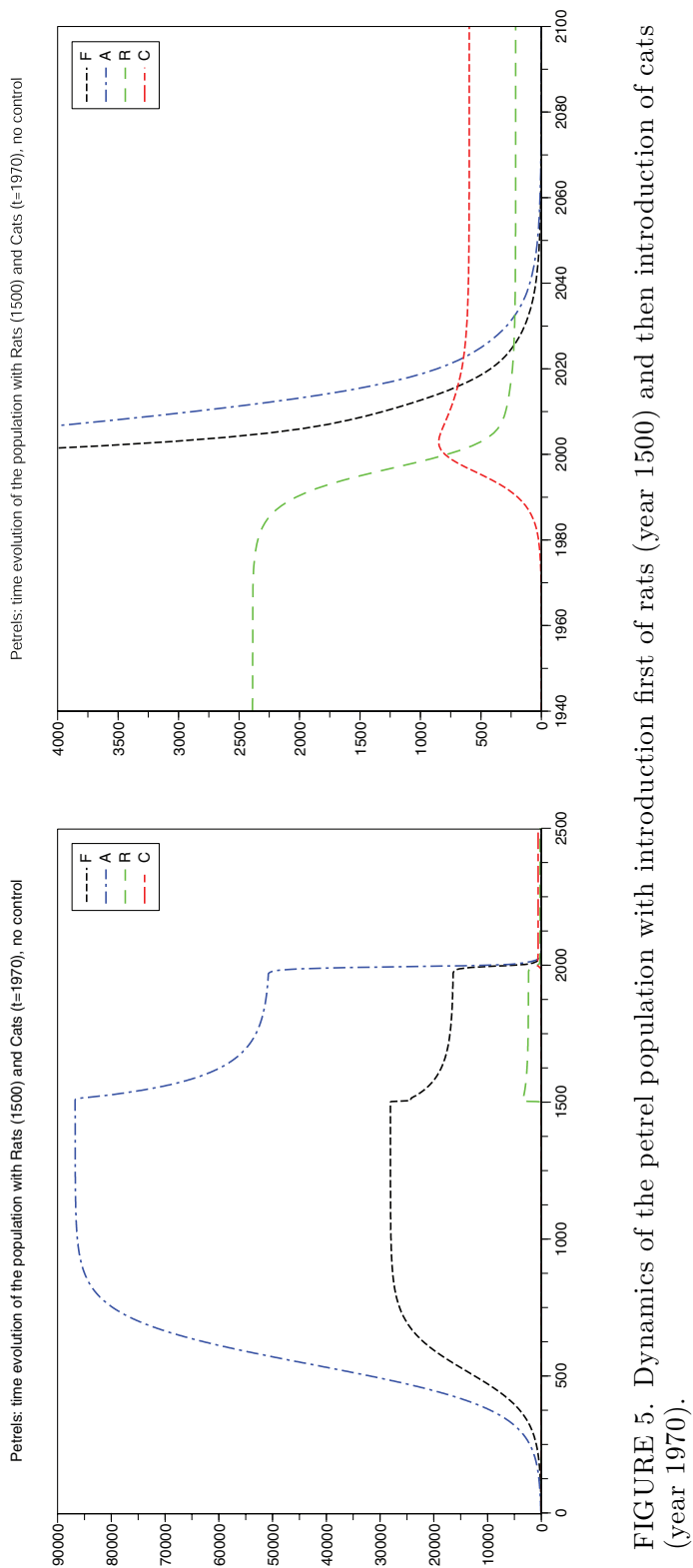


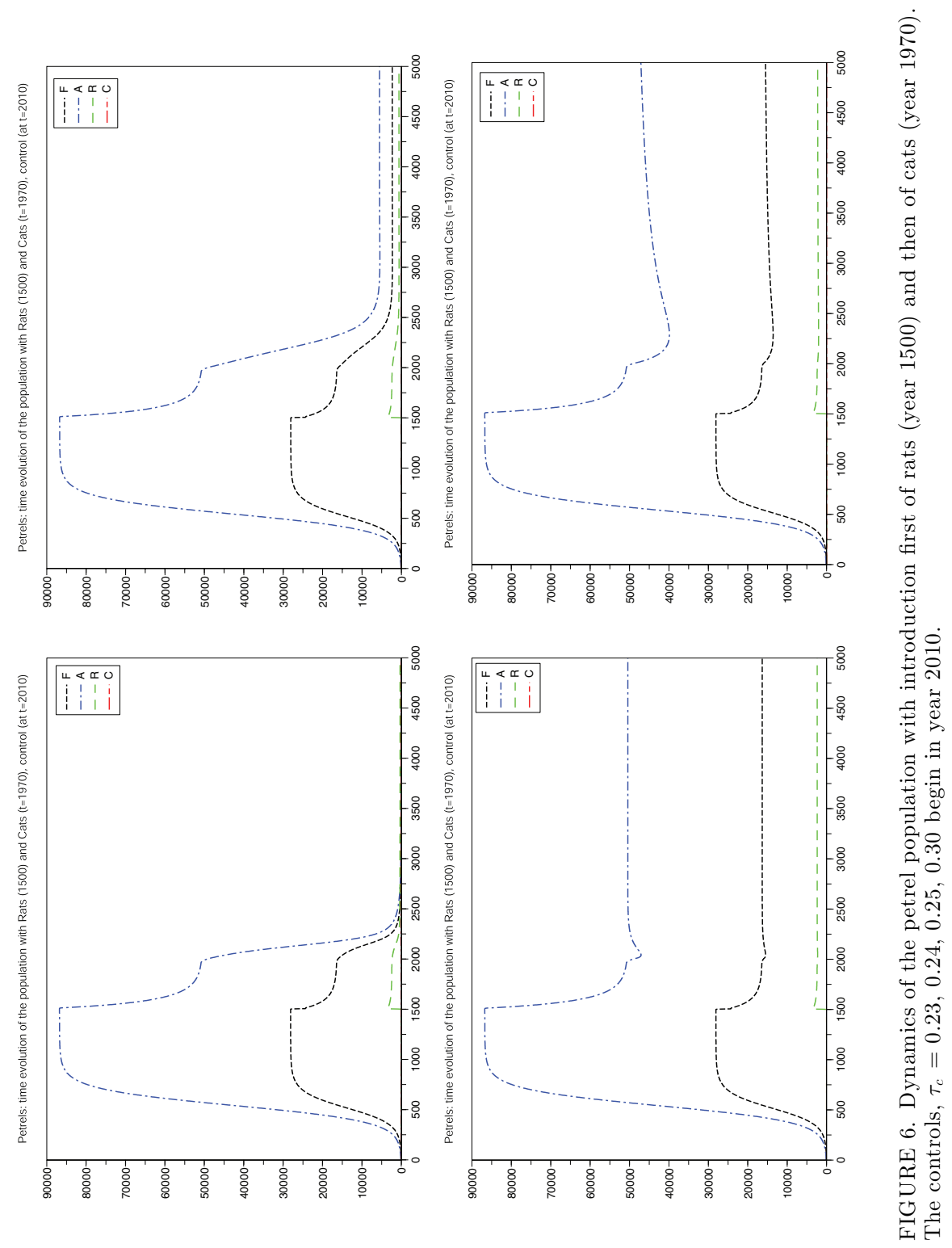


extinction will strongly depend on the control: if $\tau_{c}=0$, the petrels go extinct in a few decades, but if $\tau_{c}=0.23$ it will takes 8 centuries (see Figure 6a). Because $\left.\tau_{c} \in\right] \tau_{*}, r_{c}$ [, the system will reach equilibrium $E_{f u l l}$ : see Figure 6a with $\tau_{c}=0.24$. If $\tau_{c} \geq r_{c}$, then the system will reach the equilibrium $E^{* *}$. For instance with the values $\tau_{c}=0.25$ and $\tau_{c}=0.30$, the cats disappear and the petrel population increases again (see Figure $6 \mathrm{c}$ and d). The recovery of the petrel population is slow however, given their life-history parameters and low reproductive rate.

Finally, in Figure 7, we summarize the long-term behavior of the different populations, i.e., the different equilibria reached by the full system, in relation to the control parameter $\tau_{c}$. We also recover some of the results given in the previous sections: the system tends to one of the equilibrium given in Propositions 6 and 8. As seen in the previous figures, as long as cats are present, i.e., as long as the control is insufficient, the petrel population eventually goes extinct, i.e., the system tends to equilibrium $E^{* * *}$. If the control becomes sufficient, then we show that there is a small "window" in which it is possible to reach the full three species equilibrium $E_{f u l l}$, or when the control or the rate of capture is large enough, i.e., $\tau_{c} \geq \tau^{*}$, the petrel and rat populations reach rapidly the equilibrium $E^{* *}$. It is clear that if it is possible to maintain the cat population at a low level, the petrel population can grow "rapidly" to a substantial number of adults and fledgings. Also, the system is sensitive to the control parameter $\tau_{c}$ : small perturbations in $\tau_{c}$, around $\tau_{*}$, can drastically change the behavior of the whole system. Overall, cat control is necessary to prevent the extinction of the petrels. Even if the control is not sufficient enough, i.e., we do not eradicate all cats, it appears that even a low level of cat control can greatly delay the extinction of the petrels.

In the same manner, it is possible to consider rat control, but the impact of rats on the petrel population is very low in comparison with cats. We perform computations with different levels of rat and cat control i.e., $0 \leq \tau_{R} \leq 5$ and $0.22 \leq \tau_{c} \leq 0.26$ (Figures 8 and 9 ). As expected from theory, we show that rat control does not influence the long-term behavior of the petrel population so long as cats are present. Moreover, in Figure 8, we show that $\tau_{*}$, the minimal sufficient cat control parameter given in Proposition 6, depends on $\tau_{r}$, but its variation is rather small, showing that the impact of rat control is relatively weak in comparison with cat control. Control or even eradication of rats has 


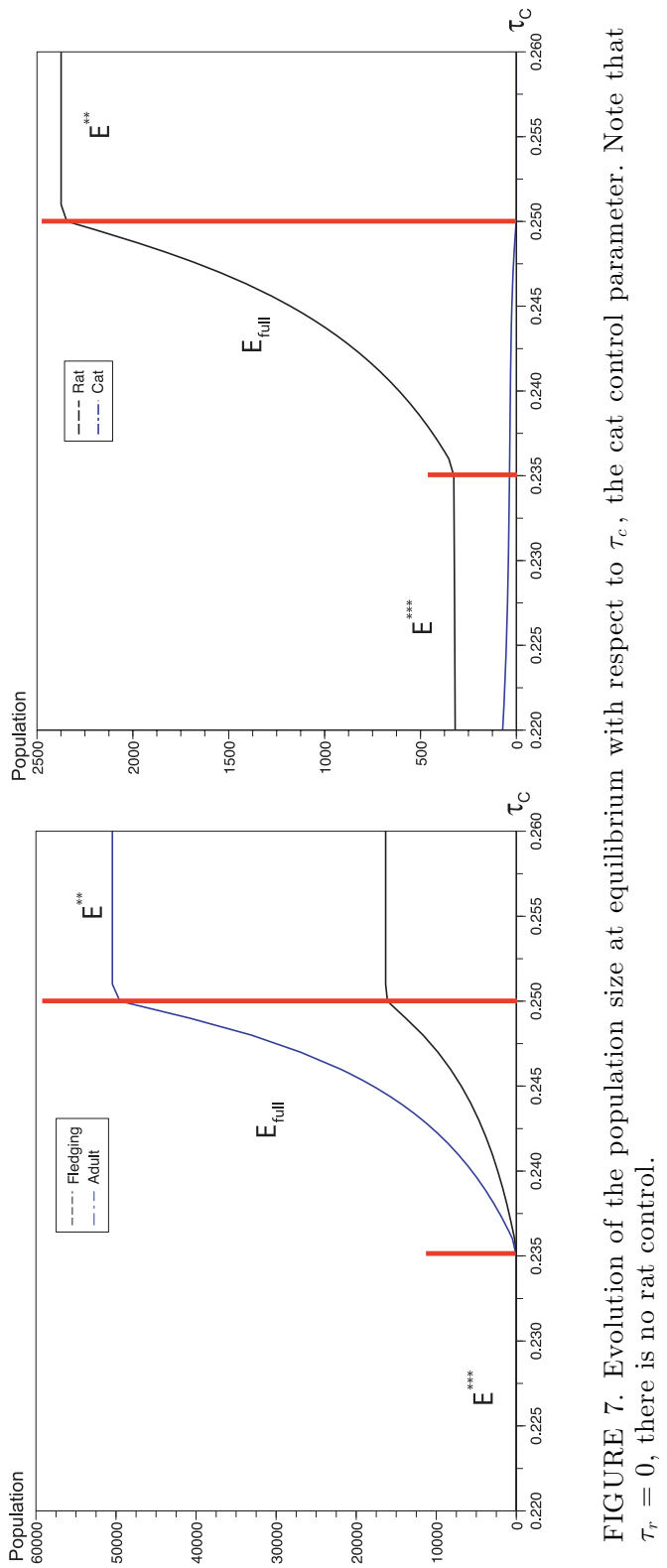




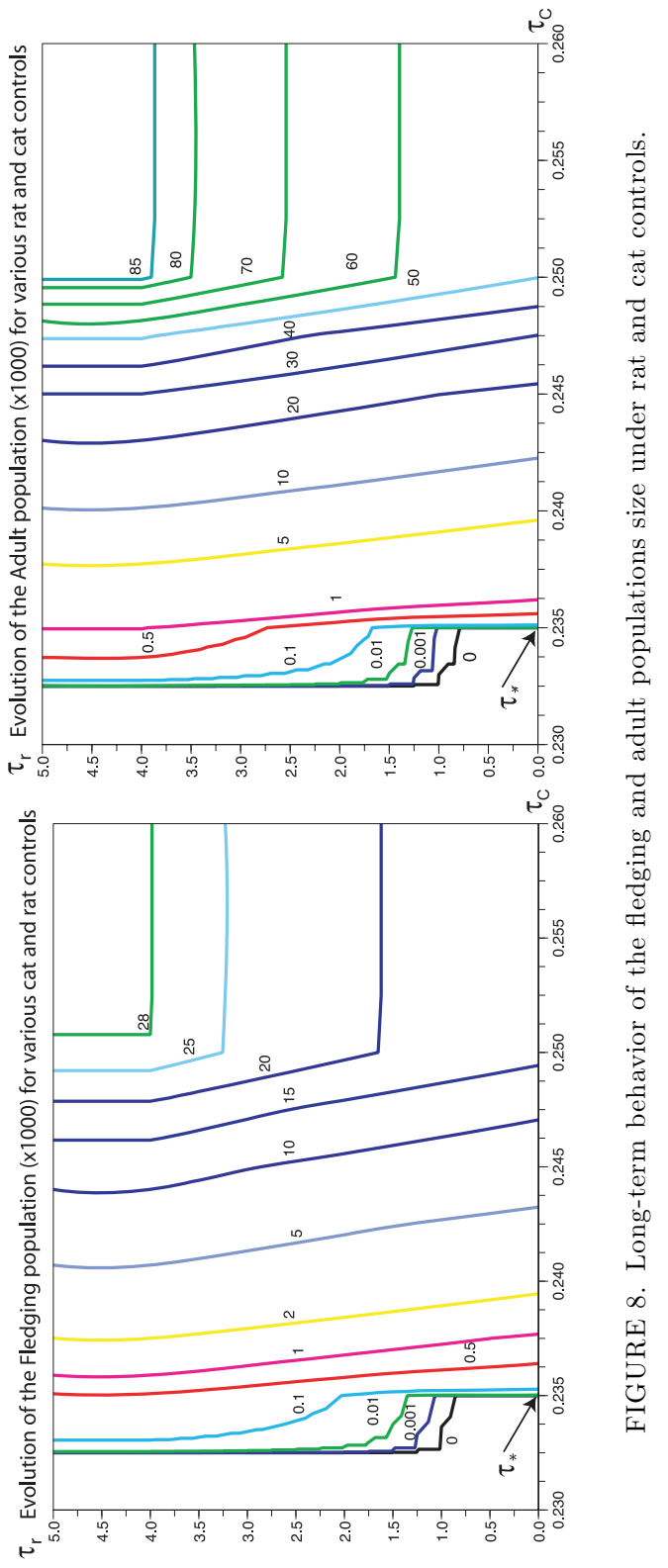




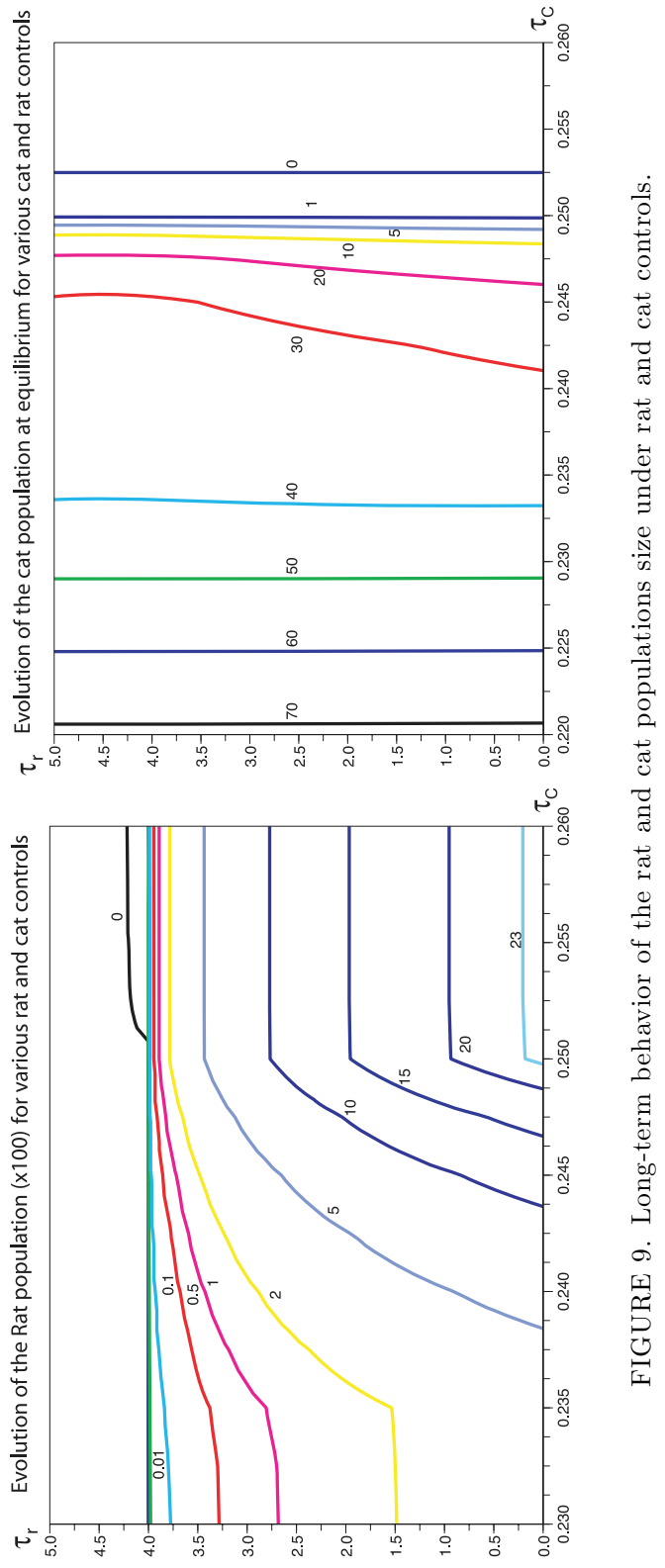


TABLE 4. Model parameterization for seasonality.

\begin{tabular}{lrc} 
& October-March & April-September \\
\hline$\gamma_{c s}=$ & 168 & 336 \\
$\gamma_{c a}=$ & 70 & 0 \\
$\gamma_{c f}=$ & 22 & 0 \\
$\gamma_{r v}=$ & 300 & 600 \\
$\gamma_{r f}=$ & 8 & 0 \\
\hline
\end{tabular}

elsewhere been shown to only delay the extinction of other mediumsized seabird species, in the presence of adult mortality (Igual et al. [2009]).

4.3. Numerical experiments with periodicity. Here, we introduce seasonality in seabird breeding by repeating the experiments given in the previous section. Indeed, we know that gadfly petrels are seasonal breeders present on colonies for only some of the year. Thus for some of the year rats (and cats) can eat eggs, fledgings, and adults. During this time the mortality induced by cats is substantial, and petrels can represent more than $50 \%$ of cat prey (Faulquier et al. [2008]). However predator swamping by the massive influx of breeding seabirds has also been hypothesized to facilitate seabird persistence, due to a presumed lag in the predator growth rate following off-season population collapse (e.g., Catry et al. [2007]; Taylor [1979]). Such an off-season collapse in cats could be compensated for by the presence of rats as an alternative food supply for cats.

Following Table 3, we alter some values in the predation rates (see Table 4). Using the same numerical scheme with $\Delta t=\frac{1}{2}$ (half a year) for the whole system, we obtain the results given in Figure 10. As expected, oscillations appear due to seasonality and, from year 1500 to year 1970, the petrel and rat populations stay stable around mean values. However, with seasonality, the impact of rats on the petrel population is lower than in the nonperiodic case (compare Figures 5 and 10). When cats are introduced in year 1970, seasonality reduces 


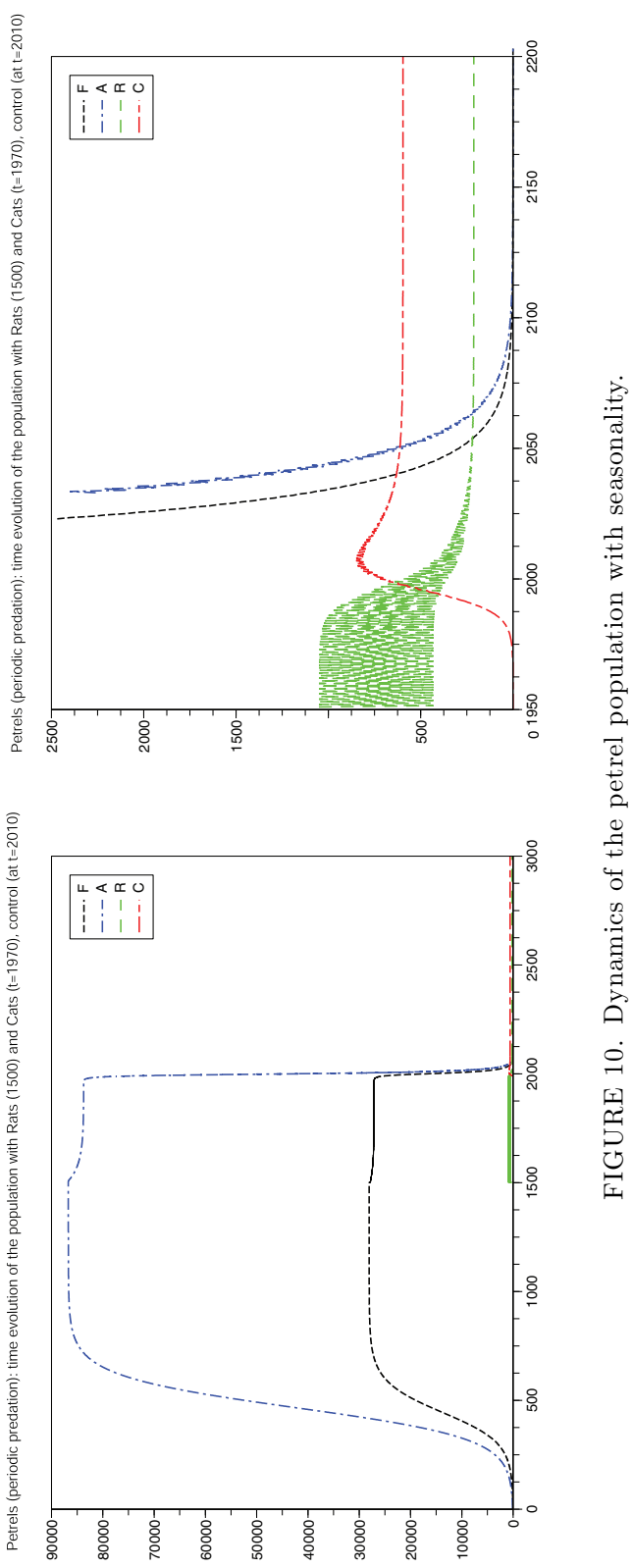


the rate of decline of the population, but the impact of the cats on the adult population remains so severe that after one and a half centuries, the petrel population still goes extinct, i.e., the long-term behavior of the system does not change. Thus overall, the same conclusion holds with or without including seasonality in the model: cats should be controlled or eradicated as the priority for seabird conservation.

5. Conclusion. We have presented an extension of previous mesopredator release models (Courchamp et al. [1999]; Fan et al. [2005]), with a particular focus on an endangered endemic seabird: the Barau's petrel in Réunion Island. Our model takes into account more biologically realistic assumptions for long-lived birds, and medium-sized seabirds in particular. This leads to a nonlinear dynamical system of nine differential equations. We provide a theoretical study of this system and demonstrate existence of a unique solution as well as the stability/instability of plausible equilibria. We address the "persistence question" of Fan et al. [2005], and except when $\left.\tau_{c} \in\right] \tau_{*}, r_{c}[$, we show that equilibrium coexistence of all three species is not possible, principally because seabirds vulnerable at all life stages can not coexist with predators. If $\left.\tau_{c} \in\right] \tau_{*}, r_{c}[$, all three species can coexist but only if superpredator control is sufficiently large but less than the predator growth rate.

In order to present reliable numerical simulations, we developed a nonstandard finite difference scheme suitable to preserve the positivity of the solution, as well as the equilibria and their qualitative properties. Our scheme is said to be dynamically consistent with the continuous problem. In that sense, it is superior to standard methods, implemented in scientific softwares like Matlab or Scilab (see for instance Anguelov et al. [2009]). We would like to point out that the choice or the construction of reliable algorithms is strongly related to a preliminary theoretical study of the considered system.

Using data obtained from field observations (Pinet et al. [2008]), we were able to parameterize our model and then to run some numerical experiments. The simulations confirmed the theoretical results and particularly showed that cats cannot protect seabirds but in fact play a major role in their extinction. We demonstrated the validity of this conclusion by example of the Barau's petrel, endemic to Réunion Island. 
Recent cat invasion on colonies is the major contributor to petrel population decline, and given the current population size ( $<6,500$ pairs), if substantial cat control is not immediately undertaken the population is likely to go extinct within the next few decades. Even with the eradication of cats, recovery of the petrel population could take many centuries. This scenario is likely to be common to most petrel species threatened by predators (e.g., Bonnaud et al. [2009]; Le Corre [2008]).

Our model was purposefully limited to demographic parameters, which are comprehensible to biologists and routinely estimated in seabird and predator population studies, such as population size, survival, mortality, and growth rates. Raw data typically come from longterm individual-based studies of population dynamics (Lebreton et al. [1992]). Per capita predation rates are difficult to study in the field, and instead the gross percentage reduction in prey survival or reproductive success is commonly measured instead. These values are difficult to transform to per capita predation rates as they depend on both predator and prey population densities. Instead, per capita predation rates can be estimated from diet analysis (Bonnaud et al. [2007]).

As we have shown, extensions to our model are possible to allow more realistic population dynamics, although we believe these are unlikely to change our qualitative results, only the time scale over which population decline and recovery might occur. Additionally, analytical solutions to more complex models will be difficult, although numerical investigation would remain possible. But, as we have shown, taking into account seasonality, at least in the case of the petrels, does not change the long-term behavior of the system. Immigration of new predators into breeding colonies could be readily incorporated into the model, representing a "vacuum" effect following local control operations. This rapid influx of predators would artificially increase the growth rate of the cat population and require a higher control effort.

Predator-prey systems involving more than two species (i.e., additional predators or prey) can behave in complex manners but are characteristic of islands invaded by introduced species (Courchamp et al. [2003]). Our simulations have focused on a dual predator single prey 
mesopredator release system, but the exact trophic role of introduced species can be flexible, particularly due to the adaptability of invasive mammals. For example, rats may function more as an alternative prey to cats rather than an additional predator of seabirds. If this were the case, the system dynamics would better characterize hyperpredation (Courchamp et al. [2000]), where the presence of an alternative food supply can maintain the key superpredator at an elevated density (e.g., Bonnaud et al. [2007]; Peck et al. [2008]). The dynamics of this model have been explored elsewhere, and also lead to prey extinction (Gaucel and Pontier [2005]). We finally emphasize that our work applies strictly to long-lived (K-selected) prey species, where age structure plays an important role in demography.

We believe our work can be applied to study the long time dynamics of other endangered seabirds, like the Mascarene petrel, Pseudobulweria aterrima, the Audubon's shearwater, Puffinus lherminieri, which is now extinct on Mauritius Island but still exists on Réunion Island, and many other seabirds around the world.

\section{APPENDiCES}

Appendix A. Very often in ecology or in epidemiology, modeling leads to problems involving matrices with special structure. Here we recall, for readers' convenience, some useful definitions and results related to nonnegative matrices, $M$-matrices, and Metzler matrices.

Let $A$ be an $n \times n$ matrix. We note $S p(A)$, the spectrum of $A, \rho(A)$ is the spectral radius and $s(A)=\{\max \operatorname{Re} \lambda ; \lambda \in \sigma(A)\}$, the stability modulus of $A$. We also use the following notations

$$
\begin{gathered}
A \leq B \Longleftrightarrow a_{i j} \leq b_{i j}, \text { for all }(i, j) . \\
A<B \Longleftrightarrow A \leq B, \text { and there exist at least one pair }\left(i_{0}, j_{0}\right) \\
\text { such that } a_{i_{0} j_{0}}<b_{i_{0} j_{0}}, \\
A \ll B \Longleftrightarrow a_{i j}<b_{i j}, \text { for all }(i, j) .
\end{gathered}
$$

Theorem 3. (Berman and Plemmons [1979], Theorem 2.1.3, p. 27) Let $A$ be a real $n \times n$ matrix with nonnegative entries. Then $\rho(A)$ is 
an eigenvalue and there exists a nonnegative eigenvector $v$ corresponding to $\rho(A)$. Furthermore, if $A$ is irreducible, then $\rho(A)$ is a simple eigenvalue and $A$ has a positive eigenvector corresponding to $\rho(A)$.

Theorem 4. (Berman and Plemmons [1979] Corollary 2.1.5, p. 27) Let $A$ and $B$ be real nonnegative $n \times n$ matrices such that $A<B$ and $A+B$ is irreducible. Then $\rho(A)<\rho(B)$.

A matrix $A$ is called a Metzler matrix if all its off-diagonal entries are nonnegative.

A matrix $A$ is called an M-matrix if it can be written in the form $A=\mu I d-B$, where $B \geq 0$ and $\mu>\rho(B)$.

In Berman and Plemmons [1979] and Fiedler [1986], the interested reader can find many useful results about $M$-matrices like the following one:

Theorem 5. Let $A \in \mathbb{R}^{n \times n}$. each of the following conditions (among others) is equivalent to the statement " $A$ is an $M$-matrix"

- All principal minors of $A$ are nonnegative.

- Every real eigenvalue of $A$ is nonnegative.

- The real part of each nonzero eigenvalue of $A$ is positive, i.e., $s(A)>0$.

Definition 1. A matrix $B$ is Metzler stable if and only if $B$ is a Metzler matrix and $s(B) \leq 0$.

Definition 2. A is a stable Metzler matrix if and only if $-A$ is an M-matrix.

Definition 3 (Regular splitting). Let $A$ be a real Metzler matrix. $A=N+M$ is a regular splitting, if $N$ is a Metzler stable matrix $M \geq 0$ is a nonnegative matrix.

Proposition 9 (Berman and Plemmons [1979]; Varga [1962]). Let $A=N+M$ be a regular splitting of $A$ a real Metzler matrix. Then $A$ is Metzler stable if and only if $\rho\left(-N^{-1} M\right)<1$. 
In particular, the previous proposition infers the following useful equivalence

$$
s(A)<0 \Longleftrightarrow \rho\left(-N^{-1} M\right)<1 .
$$

Appendix B. We first show that $T E$ is GAS.

- It suffices to consider the following Lyapunov function

$$
\begin{aligned}
V(X) & =F+\frac{1}{\sigma_{f}} J_{1}+\frac{1}{\sigma_{f} \sigma} J_{2}+\cdots+\frac{1}{\sigma_{f} \sigma^{n-1}} J_{n}+\frac{1}{\sigma_{f} \sigma^{n}} A, \\
& =\langle W, X\rangle
\end{aligned}
$$

with $W=\left(1, \frac{1}{\sigma_{f}}, \frac{1}{\sigma_{f} \sigma}, \ldots, \frac{1}{\sigma_{f} \sigma^{n-1}}, \frac{1}{\sigma_{f} \sigma^{n}}\right) \gg 0$. We easily verify that $V\left(E_{0}\right)=0$ and $V(X)>0$ for all $X \in \mathcal{G}^{-} \backslash\left\{E_{0}\right\}$. Moreover, a straighforward computation shows that

$$
\dot{V}(X)=\langle W, \mathcal{A}(X) X\rangle-\alpha \beta \delta \varepsilon\left(\frac{F}{K_{F}}+\left(\frac{1}{\mathcal{R}}-1\right)\right) A
$$

is less or equal to zero if $\mathcal{R} \leq 1$. Moreover the maximal invariant set contained in $\dot{V}=0$ is $E_{0}$. Thus, from Lyapunov theory, we deduce that $E_{0}$ is GAS.

- Using the fact that

$$
\left\{\begin{array}{c}
F^{*}=\left(1-\frac{1}{\mathcal{R}}\right) K_{f}, \\
A^{*}=\frac{1}{\alpha \beta \delta \varepsilon}(\mathcal{R}-1) K_{F},
\end{array}\right.
$$


we compute the Jacobian matrix at $E_{*}$

$$
J_{\mathcal{A}}\left(E_{*}\right)=\left(\begin{array}{ccccc}
-\mathcal{R} & 0 & & 0 & \frac{\alpha \beta \delta \varepsilon}{\mathcal{R}} \\
\sigma_{f} & -1 & \ddots & & 0 \\
0 & \ddots & \ddots & \ddots & \\
\vdots & & \ddots & -1 & \ddots \\
& & & \sigma_{J_{n}} & -\mu_{a}
\end{array}\right),
$$

which is a Metzler matrix that admits a regular splitting $J_{\mathcal{A}}\left(E_{*}\right)=$ $N+M$, with

$$
\begin{aligned}
& N=\operatorname{diag}\left(-\mathcal{R}, \ldots,-1,-\mu_{A}\right) \text { and } \\
& M=\left(\begin{array}{cccccc}
0 & 0 & \ldots & 0 & 0 & \frac{\alpha \beta \delta \varepsilon}{\mathcal{R}} \\
\sigma_{f} & 0 & 0 & \ddots & 0 & 0 \\
0 & \sigma & 0 & 0 & \ddots & 0 \\
\vdots & \ddots & \ddots & \ddots & \ddots & 0 \\
\vdots & \ddots & \ddots & \sigma & 0 & 0 \\
0 & \ldots & \ldots & 0 & \sigma & 0
\end{array}\right) .
\end{aligned}
$$

Thus, it is clear that $N$ is Metzler stable and $M \geq 0$. Moreover, we 
have

$$
-N^{-1} M=\left(\begin{array}{cccccc}
0 & 0 & \cdots & 0 & 0 & \frac{\alpha \beta \delta \varepsilon}{\mathcal{R}^{2}} \\
\sigma_{f} & 0 & 0 & \ddots & 0 & 0 \\
0 & \sigma & 0 & 0 & \ddots & 0 \\
\vdots & \ddots & \ddots & \ddots & \ddots & 0 \\
\vdots & \ddots & \ddots & \sigma & 0 & 0 \\
0 & \cdots & \cdots & 0 & \frac{\sigma}{\mu_{A}} & 0
\end{array}\right)
$$

and a straightforward computation shows that $\rho\left(-N^{-1} M\right)<1$ if $\frac{\alpha \beta \delta \varepsilon}{\mathcal{R}^{2}} \frac{\sigma_{f} \sigma^{n}}{\mu_{A}}=\frac{1}{\mathcal{R}}<1$ or equivalently $\mathcal{R}>1$. Thus from Proposition 9 and relation (A1), we deduce that $s\left(J_{\mathcal{A}}\left(E_{*}\right)\right)<0$ if $\mathcal{R}>1$ and the result follows.

Appendix C. We solve $\frac{d X}{d t}=0$ in (9). The first three equilibria are easy to obtain using the previous section. The last equilibrium $E_{*}^{r}$ is obtained through straighforward computations. Assuming $r_{r}-\tau_{r}>0$, we get

$$
\left\{\begin{array}{c}
R^{r}=\left(1-\frac{\tau_{r}}{r_{r}}\right) \frac{\left(\gamma_{v}^{r} F^{r}+\gamma_{f}^{r} V\right)}{\gamma_{f}^{r} \gamma_{v}^{r}} \\
A^{r}=\frac{\sigma}{\mu_{a}} J_{n}^{r}, \\
J_{n}^{r}=\sigma J_{n-1}^{r}, \\
\vdots \\
J_{1}^{r}=\sigma_{F} F^{r}
\end{array}\right.
$$

and

$$
F^{r}+\frac{F^{r}}{F^{r}+V} \gamma_{f}^{r} R^{r}=\alpha \beta \delta \varepsilon A^{r}\left(1-\frac{F^{r}}{K_{F}}\right) .
$$


We deduce that

$$
A^{r}=\frac{\sigma^{n} \sigma_{F}}{\mu_{a}} F^{r}
$$

Thus, from the last equalities, by linearization, we obtain the second order equation in $F^{r}$

$$
\begin{aligned}
& F^{r}+\frac{F^{r}}{F^{*}+V} \gamma_{f}^{r}\left(1-\frac{\tau_{r}}{r_{r}}\right) \frac{\left(\gamma_{v}^{r} F^{r}+\gamma_{f}^{r} V\right)}{\gamma_{f}^{r} \gamma_{v}^{r}}=\mathcal{R} F^{r}\left(1-\frac{F^{r}}{K_{F}}\right) \\
& \left(F^{r}+V\right)+\left(1-\frac{\tau_{r}}{\mu_{r}}\right) F^{r}+\frac{\gamma_{f}^{r}}{\gamma_{v}^{r}}\left(1-\frac{\tau_{r}}{r_{r}}\right) V=\mathcal{R}\left(1-\frac{F^{r}}{K_{F}}\right)\left(F^{r}+V\right) \\
& \frac{\mathcal{R}}{K_{F}}\left(F^{r}\right)^{2}+F^{r}\left(\mathcal{R}\left(\frac{V}{K_{F}}-1\right)+1+\left(1-\frac{\tau_{r}}{r_{r}}\right)\right)+\left(\mathcal{R}_{\tau_{r}}-\mathcal{R}\right) V=0 \\
& \left(F^{r}\right)^{2}+F^{r}\left(\left(V-K_{F}\right)+\left(2-\frac{\tau_{r}}{r_{r}}\right) \frac{1}{\mathcal{R}} K_{F}\right)+\left(\frac{\mathcal{R}_{\tau_{r}}}{\mathcal{R}}-1\right) K_{F} V=0 .
\end{aligned}
$$

This second order equation admits at a real positive solution if $\frac{\mathcal{R}_{\tau_{r}}}{\mathcal{R}}-1<0$, i.e., if $\mathcal{R}>\mathcal{R}_{\tau_{r}}$. Thus, we deduce that if $\mathcal{R}>\mathcal{R}_{\tau_{r}}$, we deduce

$$
\begin{aligned}
F^{r}= & \frac{1}{2}\left(\left(\left(\left(1-\left(2-\frac{\tau_{r}}{r_{r}}\right) \frac{1}{\mathcal{R}}\right) K_{F}-V\right)\right)\right. \\
& \left.+\sqrt{\left(\left(\left(1-\left(2-\frac{\tau_{r}}{r_{r}}\right) \frac{1}{\mathcal{R}}\right) K_{F}-V\right)\right)^{2}+4\left(1-\frac{\mathcal{R}_{\tau_{r}}}{\mathcal{R}}\right) K_{F} V}\right) .
\end{aligned}
$$


Appendix D. We first compute the Jacobian matrix associated with $B(X)$ in $(9)$

$$
\begin{aligned}
& J_{B}(X)
\end{aligned}
$$

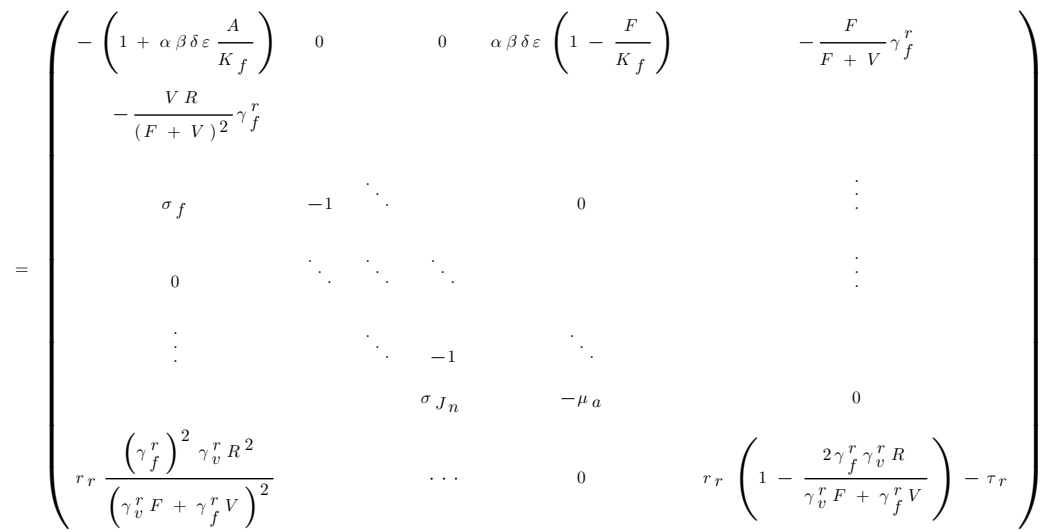

We now compute the Jacobian matrix associated with each equilibrium. We first consider

$J_{B}\left(E_{0}\right)=\left(\begin{array}{cccccc}-1 & 0 & & 0 & \alpha \beta \delta \varepsilon & 0 \\ \sigma_{f} & -1 & \ddots & & 0 & \vdots \\ 0 & \ddots & \ddots & \ddots & & \vdots \\ \vdots & & \ddots & -1 & \ddots & \\ & & & \sigma_{J_{n}} & -\mu_{a} & 0 \\ 0 & & & \ldots & 0 & r_{r}-\tau_{r}\end{array}\right)=\left(\begin{array}{cc}J_{0} & 0 \\ 0 & r_{r}-\tau_{r}\end{array}\right)$

where $J_{0}$ is the Jacobian matrix computed at the trivial equilibrium associated with the system without predators (5). We deduce that the eigenvalues of $J_{0}$ and $r_{r}-\tau_{r}$ are eigenvalues of $J_{B}\left(E_{0}\right)$. From the previous computations, we know that the eigenvalues of $J_{0}$ are strictly negative. Thus, if $r_{r}-\tau_{r}>0$, we deduce that $J_{B}\left(E_{0}\right)$ has one positive eigenvalue, which implies that $E_{0}$ is a saddle node and thus is locally 
unstable. In contrast, if $r_{r}-\tau_{r}<0$ then $E_{0}$ is locally asymptotically stable.

Then, we consider

$$
J_{\mathcal{B}}\left(E_{1}\right)=\left(\begin{array}{cccccc}
\left.-\left(1+\left(1-\frac{\tau_{r}}{r_{r}}\right) \frac{\gamma_{f}^{r}}{\gamma_{v}^{r}}\right)\right) & 0 & & 0 & \alpha \beta \delta \varepsilon & 0 \\
\sigma_{f} & -1 & \ddots & & 0 & \vdots \\
0 & \ddots & \ddots & \ddots & & \vdots \\
\vdots & & \ddots & -1 & \ddots & \\
0 & & & \sigma_{J_{n}} & -\mu_{a} & 0 \\
r_{r}\left(1-\frac{\tau_{r}}{r_{r}}\right)^{2} \gamma_{f}^{r} & & & \cdots & 0 & -\left(r_{r}-\tau_{r}\right)
\end{array}\right) .
$$

Using the same method used to prove Proposition 2, we can show that if $\mathcal{R}<1$ and $r_{r}>\tau_{r}, J_{B}\left(E_{1}\right)$ is a stable Metzler matrix, i.e., $s\left(J_{B}\left(E_{1}\right)\right)<$ 0 , which implies that $E_{1}$ is locally asymptotically stable. This is a reasonable result in the sense that the particular equilibrium $E_{1}$ should exist if and only if $\mathcal{R}<1$.

Now, we suppose that $\mathcal{R}>1$ and we study

$$
J_{\mathcal{B}}\left(E_{*}\right)=\left(\begin{array}{cccccc}
-\mathcal{R} & 0 & & 0 & \mu_{a} & -\frac{F^{*}}{F^{*}+V} \gamma_{f}^{r} \\
\sigma_{f} & -1 & \ddots & & 0 & \vdots \\
0 & \ddots & \ddots & \ddots & & \vdots \\
\vdots & & \ddots & -1 & \ddots & \\
& & & \sigma_{J_{n}} & -\mu_{a} & 0 \\
0 & & & \cdots & 0 & r_{r}-\tau_{r}
\end{array}\right) .
$$


Thus, $s\left(J_{\mathcal{B}}\left(E_{*}\right)\right)=\max \left(s\left(J_{\mathcal{A}}\left(E_{*}\right), r_{r}-\tau_{r}\right)\right)$. In Appendix B, we proved that $s\left(J_{\mathcal{A}}\left(E_{*}\right)\right)<0$ if $\mathcal{R}>1$. Thus if $\mathcal{R}>1$ and $r_{r}>\tau_{r}$ then $J_{\mathcal{B}}\left(E_{*}\right)$ has one positive eigenvalue, which implies that $E_{*}$ is locally asymptotically unstable. Finally, we study

$$
\begin{aligned}
& J_{\mathcal{B}}\left(E_{*}^{r}\right) \\
& =\left(\begin{array}{cccccc}
-\left(1+\gamma_{f}^{r} \frac{V R^{r}}{\left(F^{r}+V\right)^{2}}+\mathcal{R} \frac{F^{r}}{K_{f}}\right) & 0 & & 0 & \alpha \beta \delta \varepsilon\left(1-\frac{F^{r}}{K_{f}}\right) & -\frac{F^{r}}{F^{r}+V} \gamma_{f}^{r} \\
\sigma_{f} & -1 & \ddots & & 0 & \vdots \\
0 & \sigma & \ddots & \ddots & & \vdots \\
\vdots & & \ddots & -1 & \ddots & 0 \\
r_{r} \frac{1}{\gamma_{f}^{r}}\left(1-\frac{\tau_{r}}{r_{r}}\right)^{2} & & & \sigma & -\mu_{a} & -\left(r_{r}-\tau_{r}\right)
\end{array}\right) .
\end{aligned}
$$

Then, the characteristic polynomial gives

$$
\begin{aligned}
p_{* *}(x)= & -(-1)^{n}\left(\left(x+r_{r}-\tau_{r}\right)\left(x+1+\gamma_{f}^{r} \frac{V R^{r}}{\left(F^{r}+V\right)^{2}}+\mathcal{R} \frac{F^{r}}{K_{f}}\right)\right. \\
& \left.-r_{r}\left(1-\frac{\tau_{r}}{r_{r}}\right)^{2} \frac{F^{r}}{F^{r}+V}\right) \times\left(x+\mu_{a}\right)(x+1)^{n} .
\end{aligned}
$$

Thus, it suffices to focus on $\left(\left(x+r_{r}-\tau_{r}\right)\left(x+1+\gamma_{f}^{r} \frac{V R^{r}}{\left(F^{r}+V\right)^{2}}+\right.\right.$ $\left.\left.\mathcal{R} \frac{F^{r}}{K_{f}}\right)-r_{r}\left(1-\frac{\tau_{r}}{r_{r}}\right)^{2} \frac{F^{r}}{F^{r}+V}\right)$ and in particular to show that all coefficients are positive. After some computations, this is true if

$$
\begin{gathered}
(\mathcal{R}-1)+\frac{\tau_{r}}{r_{r}}+\left(1-\frac{\tau_{r}}{r_{r}}\right) \frac{V}{V+F^{r}}>\gamma_{f}^{r} \frac{V R^{*}}{\left(F^{r}+V\right)^{2}} \\
=\frac{V}{\left(F^{r}+V\right)^{2}}\left(1-\frac{\tau_{r}}{r_{r}}\right) \frac{\left(\gamma_{v}^{r} F^{r}+\gamma_{f}^{r} V\right)}{\gamma_{v}^{r}}
\end{gathered}
$$


or equivalently

$$
\text { (A4) } \quad(\mathcal{R}-1)+\frac{\tau_{r}}{r_{r}}>\frac{V^{2}}{\left(F^{*}+V\right)^{2}}\left(1-\frac{\tau_{r}}{r_{r}}\right)\left(\frac{\gamma_{f}^{r}}{\gamma_{v}^{r}}-1\right) \text {. }
$$

If $\mathcal{R}>\mathcal{R}_{\tau_{r}}$, we have

$$
\mathcal{R}-1>\left(1-\frac{\tau_{r}}{r_{r}}\right) \frac{\gamma_{f}^{r}}{\gamma_{v}^{r}}>\left(1-\frac{\tau_{r}}{r_{r}}\right) \frac{\gamma_{f}^{r}}{\gamma_{v}^{r}} \frac{V^{2}}{\left(F^{*}+V\right)^{2}}
$$

which implies that (A4) is always true. Thus, we deduce that: if $\mathcal{R}>$ $\mathcal{R}_{\tau_{r}}, E_{*}^{r}$ is locally asymptotically stable.

Appendix E. We are looking for equilibria $\left(F^{*}, J_{1}^{*}, \ldots\right.$, $\left.A^{*}, R^{*}, C^{*}\right)^{T}$, which leads to either $C^{*}=0$ and then we recover the equilibria obtained for the system seabird-rat, or $C^{*} \neq 0$ and the equilibria should verify the following equalities.

$$
\left\{\begin{array}{l}
C^{*}=\left(1-\frac{\tau_{c}}{r_{c}}\right)\left(\frac{A^{*}}{\gamma_{a}^{c}}+\frac{F^{*}}{\gamma_{f}^{c}}+\frac{R^{*}}{\gamma_{r}^{c}}+\frac{S}{\gamma_{s}^{c}}\right), \\
r_{r}\left(1-\frac{\gamma_{f}^{r} \gamma_{v}^{r} R^{*}}{\gamma_{v}^{r} F^{*}+\gamma_{f}^{r} V}\right)=\tau_{r}-\frac{1}{F^{*}+A^{*}+R^{*}+S} \gamma_{r}^{c} C^{*}, \\
A^{*}=\frac{\sigma}{\mu_{a}} J_{n}^{*}-\frac{A^{*}}{F^{*}+A^{*}+R^{*}+S} \frac{\gamma_{a}^{c}}{\mu_{a}} C^{*}, \\
J_{n}^{*}=\sigma J_{n-1}^{*}, \\
\vdots \\
J_{1}^{*}=\sigma_{F} F^{*}, \\
\alpha \beta \delta \varepsilon A^{*}\left(1-\frac{F^{*}}{K_{F}}\right)=F^{*}-\frac{F^{*}}{F^{*}+V} \gamma_{f}^{r} R^{*}-\frac{F^{*}}{F^{*}+A^{*}+R^{*}+S} \gamma_{f}^{c} C^{*} .
\end{array}\right.
$$


Let $F^{*}=\cdots=A^{*}=0$ and $R^{*} \neq 0$, then

$$
\begin{aligned}
& C^{*}=\left(1-\frac{\tau_{c}}{r_{c}}\right)\left(\frac{R^{*}}{\gamma_{r}^{c}}+\frac{S}{\gamma_{s}^{c}}\right), \\
& r_{r}\left(1-\frac{\gamma_{v}^{r} R^{*}}{V}\right)=\tau_{r}-\gamma_{r}^{c} \frac{1}{R^{*}+S} C^{*} .
\end{aligned}
$$

Thus

$$
\begin{gathered}
r_{r}\left(1-\frac{\gamma_{v}^{r} R^{*}}{V}\right)\left(R^{*}+S\right)=\tau_{r}\left(R^{*}+S\right)-\gamma_{r}^{c}\left(1-\frac{\tau_{c}}{r_{c}}\right)\left(\frac{R^{*}}{\gamma_{r}^{c}}+\frac{S}{\gamma_{s}^{c}}\right), \\
-\frac{r_{r} \gamma_{v}^{r}}{V}\left(R^{*}\right)^{2}+\left(r_{r}-\tau_{r}-\frac{r_{r} \gamma_{v}^{r} S}{V}+\left(1-\frac{\tau_{c}}{\mu_{c}}\right)\right) R^{*} \\
+\left(r_{r}-\tau_{r}+\frac{\gamma_{r}^{c}}{\gamma_{s}^{c}}\left(1-\frac{\tau_{c}}{r_{c}}\right)\right) S=0, \\
\left(R^{*}\right)^{2}-\left(\left(r_{r}-\tau_{r}+\left(1-\frac{\tau_{c}}{r_{c}}\right)\right) \frac{V}{r_{r} \gamma_{v}^{r}}-S\right) R^{*} \\
-\left(r_{r}-\tau_{r}+\frac{\gamma_{r}^{c}}{\gamma_{s}^{c}}\left(1-\frac{\tau_{c}}{r_{c}}\right)\right) \frac{S V}{r_{r} \gamma_{v}^{r}}=0 .
\end{gathered}
$$

Then we obtain

$$
\begin{aligned}
& \left(R^{*}-\frac{1}{2}\left(\left(r_{r}-\tau_{r}+\left(1-\frac{\tau_{c}}{r_{c}}\right)\right) \frac{V}{r_{r} \gamma_{v}^{r}}-S\right)^{2}\right. \\
& -\frac{1}{4}\left(\left(\left(r_{r}-\tau_{r}+\left(1-\frac{\tau_{c}}{r_{c}}\right)\right) \frac{V}{r_{r} \gamma_{v}^{r}}-S\right)\right)^{2} \\
& \left.\quad+4\left(\left(r_{r}-\tau_{r}+\frac{\gamma_{r}^{c}}{\gamma_{s}^{c}}\left(1-\frac{\tau_{c}}{r_{c}}\right)\right) \frac{S V}{r_{r} \gamma_{v}^{r}}\right)\right)=0,
\end{aligned}
$$


from which we deduce

$$
\begin{aligned}
R^{*}= & \frac{1}{2}\left(\left(\left(r_{r}-\tau_{r}+\left(1-\frac{\tau_{c}}{r_{c}}\right)\right) \frac{V}{r_{r} \gamma_{v}^{r}}-S\right)\right. \\
& \left.+\sqrt{\left(\left(r_{r}-\tau_{r}+\left(1-\frac{\tau_{c}}{r_{c}}\right)\right) \frac{V}{r_{r} \gamma_{v}^{r}}-S\right)^{2}+4\left(r_{r}-\tau_{r}+\frac{\gamma_{r}^{c}}{\gamma_{s}^{c}}\left(1-\frac{\tau_{c}}{r_{c}}\right)\right) \frac{S V}{r_{r} \gamma_{v}^{r}}}\right)
\end{aligned}
$$

and $C^{*}$ follows.

\section{Appendix F.}

Proposition 10. There is no biologically realistic equilibria $\left(F^{*}, J_{1}^{*}, \ldots, A^{*}, 0, C^{*}\right)$. Seabirds can not coexist with cats.

Proof. Using the first and the third equalities in (A5) with $J_{n}^{*}=$ $\sigma^{n-1} \sigma_{f} F^{*}$ and $R^{*}=0$, we obtain the following relations between $A^{*}$ and $F^{*}$

$$
\begin{aligned}
\left(A^{*}\right)^{2} & \left(\mu_{a}+\left(1-\frac{\tau_{c}}{r_{c}}\right)\right)-\sigma^{n} \sigma_{f}\left(F^{*}\right)^{2} \\
= & \sigma^{n} \sigma_{f} F^{*}\left(S+A^{*}\right) \\
& \quad-A^{*}\left(\mu_{a}\left(F^{*}+S\right)+\gamma_{a}^{c}\left(1-\frac{\tau_{c}}{r_{c}}\right)\left(\frac{F^{*}}{\gamma_{f}^{c}}+\frac{S}{\gamma_{v}^{c}}\right)\right)
\end{aligned}
$$

and

$$
\text { (A7) }\left(\frac{\gamma_{a}^{c}}{\gamma_{f}^{c}}+\mu_{a}\right) A^{*} F^{*}-\alpha \beta \delta \varepsilon\left(A^{*}\right)^{2} \frac{\gamma_{a}^{c}}{\gamma_{f}^{c}}\left(1-\frac{F^{*}}{K_{f}}\right)=\sigma^{n} \sigma_{f}\left(F^{*}\right)^{2}
$$

Thus, from (A6) and (A7), we deduce 


$$
\begin{aligned}
& \left(A^{*}\right)^{2}\left(\mu_{a}+\left(1-\frac{\tau_{c}}{r_{c}}\right)+\alpha \beta \delta \varepsilon \frac{\gamma_{a}^{c}}{\gamma_{f}^{c}}\left(1-\frac{F^{*}}{K_{F}}\right)\right) \\
& \quad+A^{*}\left(\left(\mu_{a}+\frac{\gamma_{a}^{c}}{\gamma_{v}^{c}}\left(1-\frac{\tau_{c}}{r_{c}}\right)\right) S-\left(\frac{\tau_{c}}{r_{c}} \frac{\gamma_{a}^{c}}{\gamma_{f}^{c}}+\sigma^{n} \sigma_{f}\right) F^{*}\right)=\sigma^{n} \sigma_{f} S F^{*},
\end{aligned}
$$

from which we deduce

$$
\left(A^{*}\right)^{2}\left(a-d F^{*}\right)+A^{*}\left(b-c F^{*}\right)=e F^{*}
$$

with

$$
\left\{\begin{array}{l}
a=\left(\mu_{a}+\left(1-\frac{\tau_{c}}{r_{c}}\right)\right)+\alpha \beta \delta \varepsilon \frac{\gamma_{a}^{c}}{\gamma_{f}^{c}} \\
b=\left(\mu_{a}+\frac{\gamma_{a}^{c}}{\gamma_{v}^{c}}\left(1-\frac{\tau_{c}}{r_{c}}\right)\right) S \\
c=\left(\sigma^{n} \sigma_{f}+\frac{\gamma_{a}^{c}}{\gamma_{f}^{c}} \frac{\tau_{c}}{r_{c}}\right) S \\
d=\alpha \beta \delta \varepsilon \frac{\gamma_{a}^{c}}{\gamma_{f}^{c}} \frac{1}{K_{f}} \\
e=\sigma^{n} \sigma_{f} S
\end{array}\right.
$$

Finally rewritting (A7), we obtain the following nonlinear system to solve

$$
\left\{\begin{array}{l}
\left(A^{*}\right)^{2}\left(a-d F^{*}\right)+A^{*}\left(b-c F^{*}\right)-e F^{*}=0 \\
\frac{e}{S}\left(F^{*}\right)^{2}+\left(K_{F}-F^{*}\right) d\left(A^{*}\right)^{2}-\left(\frac{b}{S}+\frac{\gamma_{c a}}{\gamma_{c v}}\left(1-\frac{\tau_{c}}{r_{c}}\right)\right) F^{*} A^{*}=0 .
\end{array}\right.
$$

It is not feasible to obtain the analytic solutions, and thus we have to approximate the solutions. Using the parameters given in Table 3, we compute numerically the roots of the previous nonlinear system. Apart from the trivial solution $(0,0)$, there is no biologically realistic solution: 
the only positive root verifies $F^{*}>K_{f}$, which is not biologically realistic.

Appendix G. After long but straighfoward computations, we obtain the Jacobian matrix associated with system (1)-(2) for all equilibrium, namely

$$
J_{\mathcal{M}}\left(E_{0}\right)=\left(\begin{array}{cccccccc}
-1 & 0 & & 0 & 0 & \alpha \beta \delta \varepsilon & 0 & \\
\sigma_{f} & -1 & 0 & \ddots & & 0 & 0 & \vdots \\
0 & \sigma & \ddots & \ddots & \ddots & 0 & & \vdots \\
\vdots & & \ddots & \ddots & -1 & \ddots & \ddots & \\
\vdots & \ddots & & 0 & \sigma & -\mu_{a} & 0 & 0 \\
0 & 0 & & \ldots & \ldots & 0 & -\left(\tau_{r}-r_{r}\right) & 0 \\
0 & 0 & \ldots & \ldots & 0 & 0 & 0 & -\left(\tau_{c}-r_{c}\right)
\end{array}\right),
$$

$$
\begin{aligned}
& J_{\mathcal{M}}\left(E_{*}\right)= \\
& \left(\begin{array}{cccccccc}
-\left(1+\alpha \beta \delta \varepsilon \frac{A^{*}}{K_{f}}\right) & 0 & \ldots & \ldots & 0 & \mu_{a} & -\frac{F^{*}}{F^{*}+V} \gamma_{f}^{r} & -\gamma_{f}^{c} \frac{F^{*}}{F^{*}+A^{*}+S} \\
\sigma_{f} & -1 & \ddots & \ddots & \ddots & & 0 & \vdots \\
0 & \ddots & \ddots & \ddots & \ddots & \ddots & & \vdots \\
\vdots & & \ddots & \sigma & -1 & \ddots & \ddots & 0 \\
0 & & & & \sigma_{J_{n}} & -\mu_{a} & 0 & -\gamma_{a}^{c} \frac{A^{*}}{F^{*}+A^{*}+S} \\
0 & 0 & & & \ldots & 0 & -\left(\tau_{r}-r_{r}\right) & 0 \\
0 & 0 & \ldots & \ldots & 0 & 0 & 0 & -\left(\tau_{c}-r_{c}\right)
\end{array}\right)
\end{aligned}
$$




$$
\begin{aligned}
& J_{\mathcal{M}}\left(E_{* * *}\right)= \\
& \begin{array}{c}
-1+\gamma_{f}^{r} \frac{R^{*}}{V} \\
+\gamma_{f}^{c} \frac{C^{*}}{R^{*}+S}
\end{array} \\
& \sigma_{f}
\end{aligned}
$$

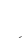

$$
\begin{aligned}
& \alpha \beta \delta \varepsilon \\
& 0 \\
& 0
\end{aligned}
$$

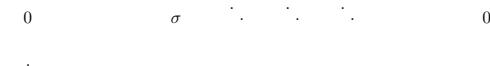

$$
\begin{aligned}
& 0 \\
& 0 \quad \sigma \quad-\left(\mu_{a}+\gamma_{a}^{c} \frac{C^{*}}{\left(R^{*}+S\right.}\right) \quad 0 \quad 0 \\
& \left.r_{r} \frac{\gamma_{v}^{r}}{\gamma_{f}^{r}} \mid \frac{R^{*}}{V}\right)^{2} \\
& 0 \quad \gamma_{c}^{r} \frac{R^{*}}{\left(R^{*}+S\right)^{2}} C^{*} \\
& -\tau_{r}-r_{r} \quad-\gamma_{r}^{c} \frac{R}{R^{*}+S} \\
& +\gamma_{c}^{r} \frac{R}{\left(R^{*}+S\right)^{2}} C^{*} \\
& \frac{r_{c}}{\gamma_{f}}\left|\frac{C^{*}}{K_{C_{*}}}\right|^{2} \\
& +2 r_{r} \gamma_{v}^{r} \frac{R^{*}}{V} \\
& \frac{r_{c}}{\gamma_{a}^{c}}\left(\frac{C^{*}}{K_{C_{*}}}\right) \\
& \frac{r_{c}}{\gamma_{r}^{c}}\left(\frac{C^{*}}{K_{C_{*}}}\right) \quad-\left(\tau_{c}-r_{c}\right. \\
& +\frac{2 r_{c} C^{*}}{K_{C} *}
\end{aligned}
$$

with $K_{C^{*}}=\frac{R^{*}}{\gamma_{r}^{c}}+\frac{S}{\gamma_{v}^{c}}$.

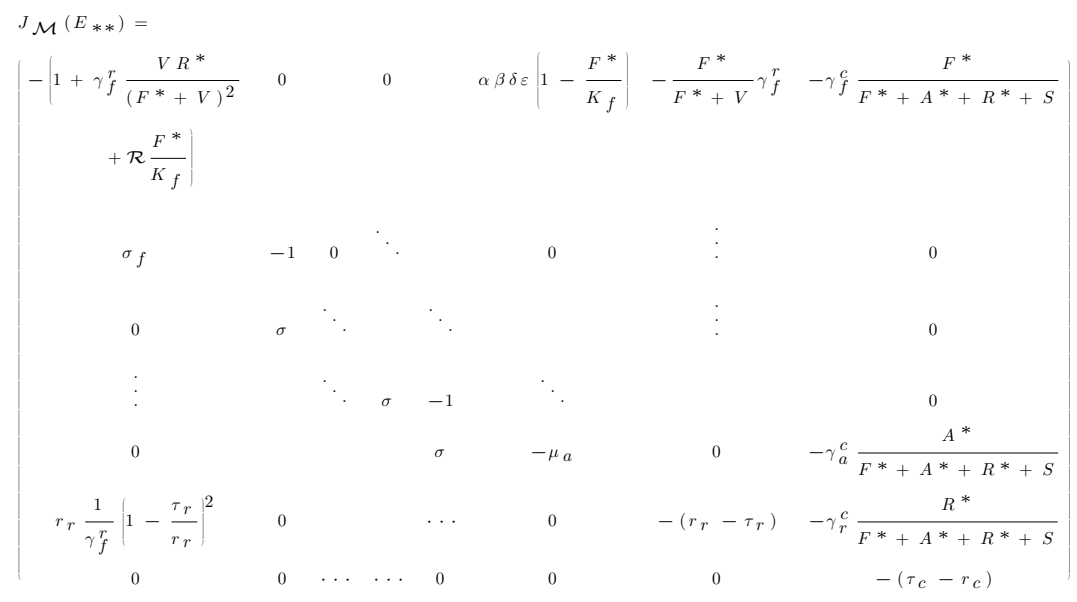

Using the results obtained in the previous appendices, we easily deduce that 
- $E_{0}$ is locally asymptotically stable if $\mathcal{R}<1, \tau_{r}>r_{r}$ and $\tau_{c}>r_{c}$.

- $E_{0}$ is unstable if $\mathcal{R}<1, \tau_{r}<r_{r}$, or $/$ and $\tau_{c}<r_{c}$.

- $E_{*}$ is unstable if $\mathcal{R}>1, \tau_{r}<r_{r}$, or /and $\tau_{c}<r_{c}$.

- $E_{*}$ is locally asymptotically stable if $\mathcal{R}>1, \tau_{r}>r_{r}$, and $\tau_{c}>r_{c}$.

From Appendix B, when $\mathcal{R}>1$, it easy to show that all eigenvalues of $J_{E_{* *}}$ are negative except one if $r_{c}-\tau_{c}>0$, which implies that $E_{* *}$ is unstable and is a saddle point. On the contrary, if $r_{c}-\tau_{c}<0$, then $E_{* *}$ is locally asymptotically stable.

Finally, for the last equilibrium $E_{* * *}$, because of the block structure of the Jacobian, we only have to focus on the following polynomial

$$
\begin{aligned}
p(x)= & \left(\tau_{r}-r_{r}+2 r_{r} \gamma_{v}^{r} \frac{R^{*}}{V}+x\right)\left(\tau_{c}-r_{c}+2 \frac{r_{c} C^{*}}{K_{C^{*}}}+x\right) \\
& +r_{c}\left(\frac{C^{*}}{K_{C^{*}}}\right)^{2} \frac{R^{*}}{R^{*}+S}
\end{aligned}
$$

If all coefficients of $p$ are strictly positive, then the roots of $p$ have negative real parts. Thus, we have to verify that

$$
2\left(r_{r} \gamma_{v}^{r} \frac{R^{*}}{V}+r_{c} \frac{C^{*}}{K_{C^{*}}}\right)>r_{r}+r_{c}-\left(\tau_{r}+\tau_{c}\right)
$$

and

$$
\begin{gathered}
\left(r_{r}-\tau_{r}\right)\left(r_{c}-\tau_{c}\right)+r_{c} \frac{C^{*}}{K_{C^{*}}} R^{*}\left(\frac{C^{*}}{K_{C^{*}}} \frac{1}{R^{*}+S}+4 r_{r} \frac{R^{*}}{V}\right) \\
>2\left(\left(r_{r}-\tau_{r}\right) r_{c} \frac{C^{*}}{K_{C^{*}}}+\left(r_{c}-\tau_{c}\right) r_{r} \gamma_{v}^{r} \frac{R^{*}}{V}\right)
\end{gathered}
$$

Using the fact that $\tau_{r}=\tau_{c}=0$ and $C^{*}=K_{C^{*}}$, the previous inequalities reduce to

$$
2 r_{r} \gamma_{v}^{r} \frac{R^{*}}{V}>r_{r}-r_{c}
$$


and

$$
\frac{R^{*}}{R^{*}+S}+r_{r} \frac{R^{*}}{V}\left(4 R^{*}-\gamma_{r v}\right)>r_{r}
$$

Then, we numerically check that these inequalities are verified with the parameters given in Table 3 . Thus, the equilibrium $E_{* *}$ is locally asymptotically stable.

\section{REFERENCES}

R. Anguelov, Y. Dumont, J. M. -S. Lubuma, and M. Shillor [2009], Comparison of Some Standard and Nonstandard Numerical Methods for the MSEIR Epidemiological Model, in (T. Simos, G. Psihoyios, Ch. Tsitouras, eds.), Proceedings of the International Conference of Numerical Analysis and Applied Mathematics, Crete, Greece, 18-22 September 2009, Volume 2, American Institute of Physics Conference Proceedings-AIP 1168, pp. 1209-1212.

A. Berman and R. J. Plemmons [1979], Nonnegative Matrices in the Mathematical Sciences, Academic Press, New York, San Francisco, London.

E. Bonnaud, K. Bourgeois, E. Vidal, Y. Kayser, Y. Tranchant, and J. Legrand [2007], Feeding Ecology of a Feral Cat Population on a Small Mediterranean Island, J. Mammal. 88, 1074-1081.

E. Bonnaud, K. Bourgeois, E. Vidal, J. Legrand, and M. Le Corre [2009], How Can the Yelkouan Shearwater Survive Feral Cat Predation? A Meta-Population Structure as a Solution? Popul. Ecol. 51, 261-270.

M. de la Brooke [1995], The Breeding Biology of the Gadfly Petrels Pterodroma spp. of the Pitcairn Islands: Characteristics, Population Sizes and Controls, Biol. J. Linnean Soc. 56, 213-231.

P. M. Catry, M. C. Silva, S. MacKay, S. Campos, J. Masello, P. Quillfeldt, and I. J. Strange [2007], Can Thin-Billed Prions Pachyptila Belcheri Breed Sucessfully on an Island with Introduced Rats, Mice and Cats? The Case of New Island, Falklands Islands, Pop. Biol. 30, 391-394.

S. Caut, E. Angulo, and F. Courchamp [2008], Dietary Shift of an Invasive Predator: Rats, Seabirds and Sea Turtles, J. Appl. Ecol. 45, 428-437.

F. Courchamp, M. Langlais, and G. Sugihara [1999], Cats Protecting Birds: Modelling the Mesopredator Release Effect, J. Anim. Ecol. 68, 282-292.

F. Courchamp, M. Langlais, and G. Sugihara [2000], Rabbits Killing Birds: Modelling the Hyperpredation Process, J. Anim. Ecol. 69, 154-164.

F. Courchamp, J.-L. Chapuis, and M. Pascale [2003], Mammal Invaders on Islands: Impact, Control and Control Impact, Biol. Rev. 78, 347-383.

D. T. Dimitrov and H. V. Kojouharov [2006], Positive and Elementary Stable Nonstandard Numerical Methods with Applications to Predator-Prey Models, J. Comp. Appl. Math. 189, 98-08.

M. Fan, Y. Kuang, and Z. Feng [2005], Cats Protecting Birds Revisited, Bull. Math. Biol. 67, 1081-1106. 
L. Faulquier, R. Fontaine, E. Vidal, M. Salamolard, and M. Le Corre [2009], Feral cats (Felis catus) threaten endangered endemic Barau's petrel Pterodroma baraui at Reunion Island (Western Indian Ocean), Waterbirds, 32, 330-336.

M. Fiedler [1986], Special Matrices and their Applications in Numerical Mathematics, Martinus Nijhoff Publishers, Dordrecht, Boston, Lancaster.

J. Gambino, M. V. Martínez-Martínez, K. Salau, E. L. Soho, D. E. Hiebeler, F. Sánchez, and D. Murillo [2007], Cats Protecting Birds Revisited with a Spatial Approach, Arizona State University, Unpublished Undergraduate Summer Project.

S. Gaucel and D. Pontier [2005], How Predator Food Preference Can Change the Destiny of Native Prey in Predator-Prey Systems? Biol. Inv. 7, 795-806.

A. B. Gumel, K. C. Patidar, and R. J. Spiteri [2005], Asymptotically Consistent Non-Standard Finite-Difference Methods for Solving Mathematical Models Arising in Population Biology. Advances in the Applications of Nonstandard Finite Difference Schemes, World Scientific Publishing, Hackensack, NJ, pp. 385421.

G. R. Howald, C. J. Donlan, J. P. Galván, J. C. Russell, J. Parkes, A. Samaniego, Y. Wang, C. R. Veitch, P. Genovesi, M. Pascal, A. Saunders, and B. Tershy [2007], Invasive Rodent Eradication on Islands, Conserv. Biol. 21(5), 1258-1268.

J. M. Igual, M. G. Forero, T. Gomez, and D. Oro [2007], Can an Introduced Predator Trigger an Evolutionary Trap in a Colonial Seabird? Biol. Conserv. 137(2), 189-196.

J. M. Igual, G. Tavecchia, S. Jenouvrier, M. G. Forero, and D. Oro [2009], Buying Years to Extinction: Is Compensatory Mitigation for Marine Bycatch a Sufficient Conservation Measure for Long-Lived Seabirds? PLoS One, 4, e4826.

H. P. Jones, B. R. Tershy, E. S. Zavaleta, D. A. Croll, B. S. Keitt, M. E. Finkelstein, and G. R. Howald [2008], Severity of the Effects of Invasive Rat on Seabirds: A Global Review, Conserv. Biol. 22(1), 16-26.

W. B. King [1985], Island Birds: Will the Future Repeat the Past? in (Moors, P. J. ed.), Conservation of Island Birds, ICBP Technical Publication 3, Cambridge, pp. 3-15.

J.-D. Lebreton, K. P. Burnham, J. Clobert, and D. R. Anderson [1992], Modeling Survival and Testing Biological Hypotheses using Marked Animals: A Unified Approach with Case-Studies, Ecol. Monogr. 62(1), 67-118.

M. Le Corre [2008], Conservation Biology: Cats, Rats and Seabirds, Nature 451, $134-135$.

M. Le Corre, A. Ollivier, S. Ribes, and P. Jouventin [2002], Light-Induced Mortality of Petrels: A Four Year Study from Réunion Island (Indian Ocean), Biol. Conserv. 105, 93-102.

R. E. Mickens [1994], Nonstandard Finite Difference Models of Differential Equations, World Scientific, Singapore.

R. E. Mickens [2000], Applications of Nonstandard Finite Difference Schemes, World Scientific, Singapore.

R. E. Mickens [2002], Nonstandard Finite Difference Schemes for Differential Equations, J. Diff. Equ. Appl. 8, 823-847.

R. E. Mickens (ed.) [2005], Advances in the Applications of Nonstandard Finite Difference Schemes, World Scientific, Singapore. 
C. B. Müller and J. Brodeur [2002], Intraguild Predation in Biological Control and Conservation Biology, Biol. Control 25, 216-223.

S. M. Moghadas, M. E. Alexander, B. D. Corbett, and A. B. Gumel [2003], Positivity-Preserving Mickens-Type Discretization of an Epidemic Model. Dedicated to Professor Ronald E. Mickens on the Occasion of his 60th Birthday, J. Diff. Equ. Appl. 9(11), 1037-1051.

M. Nogales, A. Martín, B. R. Tershy, C. J. Donlan, C. R. Veitch, N. Puerta, B. Wood, and J. Alonso [2004], A Review of Feral Cat Eradication on Islands, Conserv. Biol. 18(2), 310-319.

S. Pearre and R. Maass [1998], Trends in the Prey Size-Based Trophic Niches of Feral and House Cats (Felis catus) L., Mammal Rev. 28, 125-139.

D. R. Peck, L. Faulquier, P. Pinet, S. Jaquemet, and M. Le Corre [2008], Feral Cat Diet and Impact on Sooty Terns at Juan de Nova Island, Mozambique Channel, Anim. Conserv. 11(1), 1-10.

P. Pinet, M. Salamolard, J.-M. Probst, J. C. Russell, S. Jaquemet, and M. Le Corre [2008], Barau's Petrel (Pterodroma baraui): History, Biology and Conservation of an Endangered Endemic Petrel, Marine Ornithology, 37, 107-114.

J.-M. Probst, M. Le Corre, and C. Thibaud [2000], Breeding Habitat and Conservation Priorities in Pterodroma baraui, an Endangered Gadfly Petrel of the Mascarene Archipelago, Biol. Conserv. 93, 135-138.

S. A. Robertson [2008], A Review of Fercal Cat Control, J. Feline Med. Surg. 10, $366-375$

J. C. Russell, V. Lecomte, Y. Dumont, and M. Le Corre [2009], Intraguild Predation and Mesopredator Release Effect on Long-Lived Prey, Ecol. Model. 220(8), 1098-1104.

T. R. Simons [1984], A Population Model of the Endangered Hawaiian DarkRumped Petrel, J. Wildl. Manage. 48(4), 1065-1076.

R. H. Taylor [1979], Predation of Sooty Terns at Raoul Island by Rats and Cats, Notornis 26, 1050-1061.

R. Varga [1962], Matrix Iterative Analysis, Prentice-Hall, Prentice Hall, Englewoods Cliffs, NJ.

P. M. Vitousek, H. A. Mooney, J. Lubchenco, and J. M. Melillo [1997], Human Domination of Earth's Ecosystems, Science 277, 494-499. 